\title{
Graph Neural Network and Spatiotemporal Transformer Attention for 3D Video Object Detection from Point Clouds
}

\author{
Junbo Yin, Jianbing Shen, Senior Member, IEEE, Xin Gao, David Crandall, and Ruigang Yang
}

\begin{abstract}
Previous works for LiDAR-based 3D object detection mainly focus on the single-frame paradigm. In this paper, we propose to detect 3D objects by exploiting temporal information in multiple frames, i.e., the point cloud videos. We empirically categorize the temporal information into short-term and long-term patterns. To encode the short-term data, we present a Grid Message Passing Network (GMPNet), which considers each grid (i.e., the grouped points) as a node and constructs a $k$-NN graph with the neighbor grids. To update features for a grid, GMPNet iteratively collects information from its neighbors, thus mining the motion cues in grids from nearby frames. To further aggregate the long-term frames, we propose an Attentive Spatiotemporal Transformer GRU (AST-GRU), which contains a Spatial Transformer Attention (STA) module and a Temporal Transformer Attention (TTA) module. STA and TTA enhance the vanilla GRU to focus on small objects and better align the moving objects. Our overall framework supports both online and offline video object detection in point clouds. We implement our algorithm based on prevalent anchor-based and anchor-free detectors. The evaluation results on the challenging nuScenes benchmark show the superior performance of our method, achieving 1st on the leaderboard without any bells and whistles, by the time the paper is submitted.
\end{abstract}

Index Terms-Point Cloud, 3D Video Object Detection, Autonomous Driving, Graph Neural Network, Transformer Attention.

\section{INTRODUCTION}

$\mathrm{T}$ HE past several years have witnessed an explosion of interests in $3 \mathrm{D}$ object detection due to its vital role in autonomous driving perception. Meanwhile, the LiDAR sensor is becoming an indispensable instrument in 3D perception for its capacity of providing accurate depth information in intricate scenarios such as dynamic traffic environments and adverse weather conditions. The majority of 3D object detectors are dedicated to detecting in single-frame LiDAR point clouds, i.e., predicting oriented $3 \mathrm{D}$ bounding boxes via frame-byframe paradigm. However, little work has been devoted to detecting in multi-frame point clouds sequences, i.e., point cloud videos. In the newly released nuScenes [2] dataset, a point cloud video is defined by a driving scene containing around 400 point cloud frames captured within 20 seconds. In general, point cloud videos are readily available in practical applications and can provide rich spatiotemporal coherence. For example, object point clouds from a single frame may be truncated or sparse due to occlusions or long-distance

- J. Yin is with School of Computer Science, Beijing Institute of Technology, Beijing, China. (Email: yinjunbo@bit.edu.cn)

- J. Shen is with Inception Institute of Artificial Intelligence, Abu Dhabi, UAE. (Email: shenjianbingcg@gmail.com)

- X. Gao is with Computer, Electrical, and Mathematical Sciences and Engineering (CEMSE) Division, King Abdullah University of Science and Technology (KAUST), Thuwal, Saudi Arabia.

- D. Crandall is with the School of Informatics, Computing, and Engineering, Indiana University, Bloomington, IN 47408. (Email: djcran@indiana.edu)

- R. Yang is with the University of Kentucky, Lexington, KY 40507. (Email: ryang@cs.uky.edu)

- A preliminary version of this work has appeared in CVPR 2020 [1].

- Corresponding author: Jianbing Shen

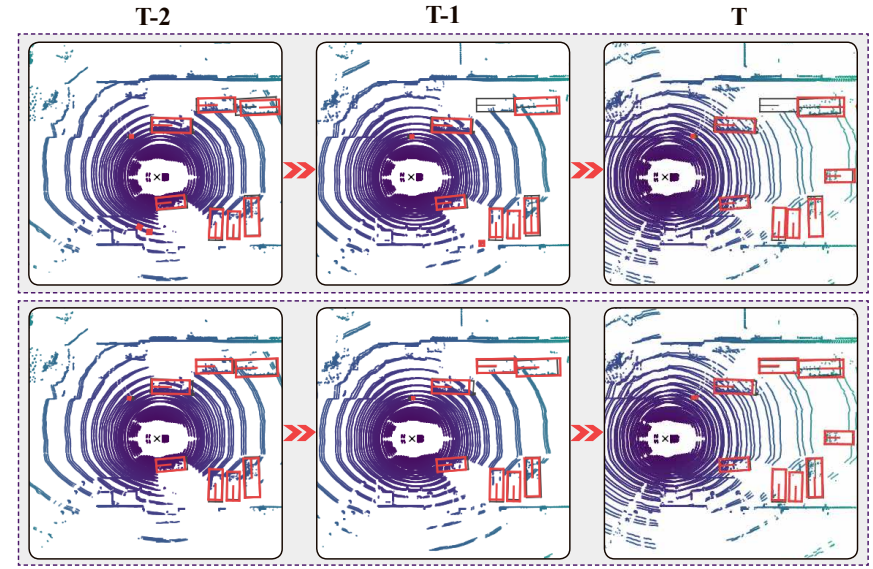

Fig. 1: A representative challenging case in autonomous driving scenarios. A typical single-frame 3D object detector, eg. [3], leads to false-negative (FN) results due to occlusion (top row). In contrast, our 3D video object detector could address this (bottom row). The red and grey boxes denote the predictions and ground-truths, respectively.

sampling, while other frames could contain complementary information for recognizing the object. Therefore, a single-frame $3 \mathrm{D}$ object detector suffers from deteriorated performance in comparison with a 3D video object detector (See Fig. 1).

Existing 3D video object detectors typically exploit the temporal information in a straightforward way, i.e., directly concatenating the point clouds from other frames to a reference frame and then performing detection in the reference frame $[3,4,5]$. This converts the video object detection task to a single-frame object detection task. However, such a simple approach has several limitations. First, in driving 
scenarios, there will be serious motion blur that will result in inaccurate detection results. One solution might be to apply ego-motion transformation for these frames. Though it can alleviate the ego-motion, it is not capable of remedying the motion blur caused by moving objects. Second, this concatenation-based approach usually simply encodes the temporal information by an additional point cloud channel such as timestamp, which ignores the rich feature relations among different frames. Third, as the label information is only provided in the reference frame, such methods will suffer more information loss when leveraging long-term point cloud data. Apart from the concatenation-based approach, other works [6] apply temporal 3D convolutional networks on point cloud videos. Nevertheless, they will encounter temporal information collapse when aggregating features over multiple frames [7].

How to effectively exploit the temporal information in point cloud videos remains an open challenge. We argue that there are two forms of temporal information, i.e., short-term and long-term patterns. Taking the nuScenes [2] dataset for example, the short-term pattern is defined by point cloud sequences captured within 0.5 seconds, including around 10 frames. As for the long-term pattern, we refer to the point cloud frames collected in 1 to 2 seconds involving dozens of frames. In this work, we aim to present a more effective and general 3D video object detection framework by enhancing prevalent 3D object detectors with both the short-term and long-term temporal cues. In particular, the long-term point clouds are first divided into several short-term ones. We separately encode each short-term data with a short-term encoding module, and then adaptively fuse the output features by a long-term aggregation module.

For handling the short-term point cloud data, our shortterm encoding module follows the paradigm of modern gridbased detectors [3, 8, 5, 9] that directly concatenate point clouds from nearby frames to a reference frame, but adopts a novel grid feature encoding strategy that models the relations of girds in nearby frames. More precisely, these detectors typically divide the point clouds into regular girds such as voxels and pillars, with each gird containing a fixed number of point clouds. Then PointNet-like modules (e.g., the Voxel Feature Encoding Layer in [8] and the Pillar Feature Network in [3]) are used to extract the grid-wise feature representation. A potential problem of these PointNet-like modules is that they only focus on the individual grid, which ignores the relations among different grids. Intuitively, certain grids in nearby frames may encode the same object parts, which can be explored to improve the detection accuracy. To this end, we propose a graph-based network, Grid Message Passing Network (GMPNet), which iteratively propagates information over different grids. GMPNet treats each non-empty grid as a graph node and builds a $k$-NN graph with the nearby $k$ grids. The relations among these grids as viewed edges. The core idea of GMPNet is to iteratively update the node features via the neighbors along the edges and hence mine the rich relations among different grid nodes. At each iteration, a node receives pair-wise messages from its neighbors, aggregates these messages and then updates its node features. After several iterations, messages from distant grids can be accessed. This flexibly enlarges the receptive field in a non-Euclidean manner. Compared with previous PointNet-like modules, GMPNet effectively encourages information propagation among different grids, meanwhile capturing short-term motion cues of objects.

After obtaining individual features extracted by the shortterm encoding module, we then turn to Convolutional Gated Recurrent Unit networks (ConvGRU [10]) to further capture the dependencies over these features in our long-term aggregation module. ConvGRU has shown promising performance in the $2 \mathrm{D}$ video understanding field. It adaptively links temporal information over input sequences with a memory gating mechanism. In each time step, the current memory is computed by considering both the current input and the previous memory features. The updated memory is then employed to perform the present task. However, there are two potential limitations when directly applying ConvGRU on multi-frame point cloud sequences. First, modern grid-based detectors tend to transform the point cloud features into the bird's eye view, and the resultant object resolution is much smaller than that in 2D images. For example, with a grid size of $0.25^{2} \mathrm{~m}^{2}$ and stride of 8 , objects such as pedestrians and vehicles just occupy around 1 to 3 pixels in the output feature maps. Therefore, when computing the current memory features, the background noise will be inevitably accumulated and decrease the detection performance. Second, the spatial features of the current input and the previous memory are not well aligned across frames. Though we could use the ego-pose information to align the static objects over multiple point cloud frames, the moving objects still incur motion blur that impairs the quality when updating the memory.

To address these challenges, we present Attentive Spatiotemporal Transformer GRU (AST-GRU), which enhances the vanilla ConvGRU with a Spatial Transformer Attention (STA) module and a Temporal Transformer Attention (TTA) module. In particular, the STA module is derived from [11, 12] and is devised to attend the small objects with the spatial context information. It acts as an intra-attention mechanism, where both the keys and queries reside in the input features. STA views each pixel as a query and the neighbors as keys. By attending the query with the context keys, STA can better tell foreground pixels from the background ones, and thus emphasize the meaningful objects in current memory features. Furthermore, we also describe a TTA module motivated by [13, 14], which is employed to align the moving objects. It is composed of modified deformable convolutional layers and behaves as an inter-attention that operates on both the input and previous memory features. TTA treats each pixel in the previous memory as a query and decides the keys by integrating the motion information, thus capturing more cues of moving objects. In contrast to the vanilla ConvGRU, the presented AST-GRU effectively improves the quality of the current memory features and leads to better detection accuracy, which is verified on both prevalent anchor-based and anchor-free detectors. Moreover, this also introduces a general methodology that accounts for both online and offline $3 \mathrm{D}$ video object detection by flexibly deciding the inputs, e.g., using previous or later frames in a point cloud video. In the offline mode, we further augment our AST-GRU with a bi- 
directional feature learning mechanism, which achieves better results in comparison to the online mode.

To summarize, the main contributions of this work are as follows:

- A general point cloud-based 3D video object detection framework is proposed by leveraging both short-term and long-term point clouds information. The proposed framework can easily integrate prevalent 3D object detectors in both online and offline modes, which provides new insights to the community.

- We present a novel graph neural network, named GMPNet, to encode short-term point clouds. GMPNet can flexibly mine the relations among different grids in nearby frames and thus capture the motion cues.

- To further model the long-term point clouds, an ASTGRU module is introduced to equip the conventional ConvGRU with an attentive memory mechanism, where a Spatial Transformer Attention (STA) and a Temporal Transformer Attention (TTA) are devised to mine the spatiotemporal coherence of long-term point clouds.

- We build the proposed 3D video object detection framework upon both anchor-based and anchor-free 3D object detectors. Extensive evaluations on the large-scale nuScenes benchmark show that our model outperforms all the state-of-the-art approaches on the leaderboard.

This work significantly extends our preliminary conference paper [1] and the improvements are multi-fold. Our previous work [1] only supports 3D object detection in the online mode with an anchor-based detector. In this work, we first provide a more general framework that works with both anchorbased and anchor-free detection settings. In this regard, our approach can be easily incorporated with leading 3D object detectors. Second, we extend our method to both online and offline detection modes, which can benefit more applications with improved accuracy, such as acting as annotation tools. Third, this paper provides a more in-depth discussion on the algorithm with more details including its motivation, technical preliminary, network architecture and implementation. Fourth, extensive ablation studies are conducted to thoroughly and rigorously assess the broad effectiveness of our model. Last but not least, we empirically observe that the proposed model outperforms all the algorithms on the nuScenes leaderboard without any bells and whistles.

\section{Related Work}

3D Object Detection. A considerable amount of efforts have been made into 3D object detection in recent years for its crucial role in autonomous driving. Existing approaches for 3D object detection can be roughly grouped into three categories. (1) $2 \mathrm{D}$ image-based methods typically perform detection from monocular $[15,16,17]$ or stereo images $[18,19,20]$. These approaches often experience great difficulty in getting promising performance due to the information loss of depth. They often turn to geometric priors [21] or integrate an additional module to estimate depth [16] or disparity [18]. It is worth noting that Wang et al. [20] presented a novel framework by first converting image-based depth maps to pseudo point clouds and then applying the off-the-shelf LiDAR-based detectors. (2) $3 D$ point cloud-based methods can leverage the LiDAR sensor to access the accurate depth information, and are less sensitive to different illumination and weather conditions. Grid- and Pointbased methods are the main ways to process the point clouds. The common solution of grid-based methods [8, 22, 3, 1] is to first discretize the raw point clouds into regular girds (e.g., voxels [9] or pillars [3]). Then 3D or 2D convolutional networks can be readily applied to extract the features. In practice, grid-based approaches are much more efficient than point-based methods, but may suffer from information loss in the quantification process. Point-based methods [23, 24, 25] typically extract features and predict proposals from pointlevel with backbones like PointNet++ [26]. They are prone to get better performance than the grid-based methods, but are limited to computational efficiency and memory footprint when the number of point clouds increases. Recently, Shi et al. [27] proposed PV-RCNN detector that combines the merits of both the voxel- and point-based approaches and shows better performance. (3) Fusion-based methods exploit both the camera and LiDAR sensors to capture complementary information. MV3D [28] is the seminal work of this family. It takes LiDAR bird's eye view and front view as well as images as inputs, and combines the proposal-wise features of each view with a deep fusion network. Later, 3D-CVF [29] proposed to combine point clouds and images from $\mathrm{N}$ multi-view cameras with a cross-view spatial feature fusion strategy. However, the multi-sensor fusion system may not work robustly due to signal synchronization problems. In this work, we focus on the LiDAR-only grid-based approaches since they are more prevalent in current autonomous driving applications.

Spatiotemporal Models in Point Clouds. Different from the aforementioned works that only perform frame-by-frame detections, we aim to address the multiple-frame 3D object detection by exploiting the temporal information. Only a few works have explored the spatiotemporal model in point clouds. Luo et al. [6] utilized temporal 3D ConvNet to aggregate the multi-frame point clouds. It performs multiple tasks simultaneously including detection, tracking and motion prediction, but leads to a considerable amount of parameters for operating on 4D tensors. Later, Choy et al. [30] improved the convolutional layers in [6] with sparse operations. However, it encounters the feature collapse issue when downsampling the features in the temporal domain, and fails to leverage full label information in all frames. More recently, [31] proposed a contemporary work with ours that focuses on a point-based recurrent gating mechanism. It adopts a Sparse Conv U-Net to extract the spatial features of each frame, and then applies LSTM on points with high semantic scores to fuse information across frames and save computation. Due to the restriction of the backbone, it is not able to be adapted to the stateof-the-art single-frame detectors. On the contrary, our work can be easily integrated into prevalent grid-based 3D object detectors [3, 5] and offers the ability to handle long-term spatiotemporal features in point cloud videos.

Graph Neural Networks. The concept of Graph Neural Networks (GNNs) was first proposed by Scarselli et al. [32]. They extended the Recursive Neural Networks (RNN) and used 
Short-term Encoding Module

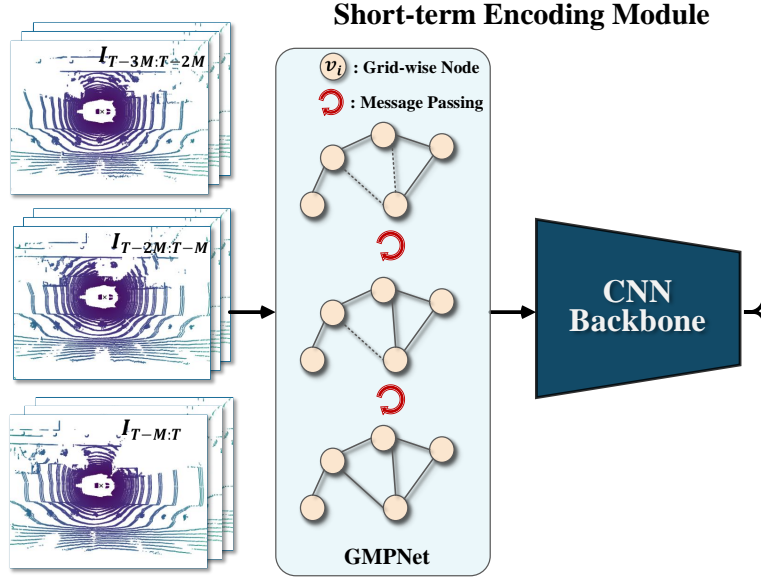

Long-term Aggregation Module

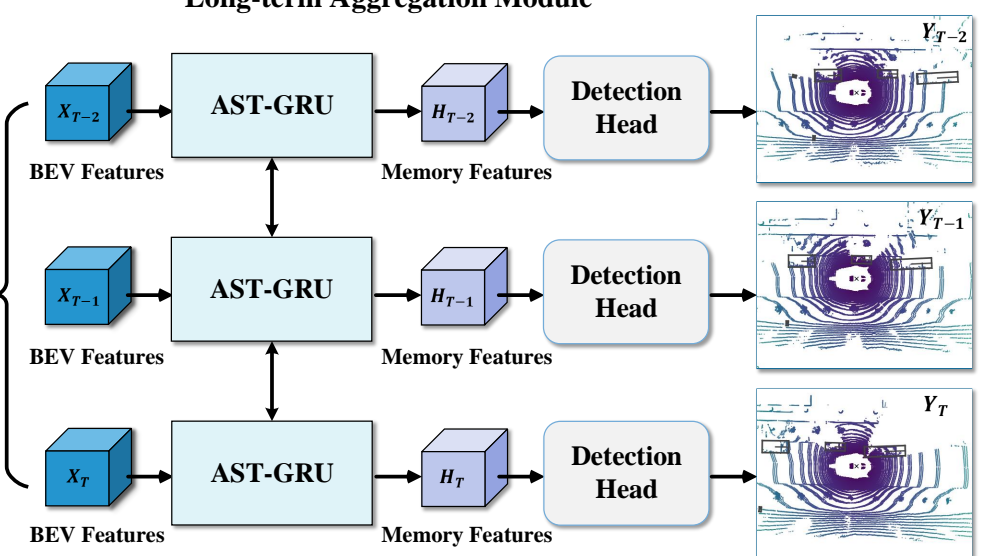

Fig. 2: Schematic of our point cloud-based 3D video object detection framework. At the time $T$, the previous $N \times M$ frames are first divided into $N$ short-term data with each merging $M$ frames. Then, the short-term encoding module extracts the BEV features for each short-term data with a Grid Message Passing Network (GMPNet) followed by a CNN backbone. Afterwards, the long-term aggregation module further captures the dependencies in these short-term features with an Attentive Spatiotemporal Transformer GRU (AST-GRU). Finally, the detection head receives the enhanced memory features and produces the detection results.

GNNs to directly process the graph-structured data. It encodes the underlying relationships among the nodes of the graph and mines the rich information in the data processing step. Afterwards, several variants have been developed to improve the representation capability of the original GNNs. Here, we categorize them into two classes according to the type of the information propagation step: convolution and gate mechanism. Graph Convolutional Networks (GCNs) [33, 34, 35, 36, 37] belongs to the former group that generalize convolution to the graph data and update nodes via stacks of graph convolutional layers. GCNs typically compute in the spectral domain with graph Fourier transformation. Recently, various applications $[38,39,40]$ have been explored by GCNs, which achieve promising performance. The latter group [41, 42, 43, 44] aimed to adopt recurrent gating units to propagate and update information across nodes. For instance, Li et al. [41] exploited the Gated Recurrent Unit (GRU) to describe the node state by aggregating messages from neighbors. After that, Gilmer et al. [45] proposed a generalized framework that formulates the graph computation as a parameterized Message Passing Neural Network (MPNN). MPNN has been well demonstrated the ability in various tasks that involve graph data [46, 47, 48]. Our GMPNet is also inspired by MPNN that encodes the features of short-term point clouds and mines the motion cues among the grid-wise nodes by iterative message passing.

\section{The Proposed 3D Video Object De- TECTION FRAMEWORK}

In this section, we first present the overall pipeline of our method in $\$ 3.1$. Then, we briefly review the general formulations of Message Passing Neural Network [45] and ConvGRU [10] in $\S 3.2$ and $\S 3.3$, respectively, since our work mainly builds upon these works. Afterwards, the crucial designs of Grid Message Passing Network (GMPNet) and Attentive Spatiotemporal Transformer GRU (AST-GRU) are described in $\S 3.4$ and $\S 3.5$, respectively. Notice that the main part of this paper follows the online object detection pipeline. While in $\S 3.6$, an offline pipeline is also presented by learning with bi-directional ConvGRU, which further improves the detection performance. Finally, we provide more detailed information on our network architecture in $\S 4.2$.

\subsection{Overview}

As illustrated in Fig. 2, our framework consists of a short-term encoding module and a long-term aggregation module. Assuming that the input long-term point cloud sequence $\left\{\boldsymbol{I}_{t}\right\}_{t=1}^{T \times M}$ contains $T \times M$ frames in total, we first divide it into multiple short-term clip $\left\{\boldsymbol{I}_{t}\right\}_{t=1}^{T}$, with each $\boldsymbol{I}_{t}$ containing concatenated point clouds within $M$ nearby frames (eg., $M=10$ in nuScenes). Then, LiDAR pose information is leveraged to align point clouds over frames in $\left\{\boldsymbol{I}_{t}\right\}_{t=1}^{T}$ to eliminate the influence of ego-motion. Next, each $\boldsymbol{I}_{t}$ is quantized into evenly distributed grids, and forwarded to the short-term encoding module to extract the bird's eye view features $\left\{\boldsymbol{X}_{t}\right\}_{t=1}^{T}$. Here, GMPNet is applied before the 2D CNN backbone to capture the relations among grids. After that, in the long-term aggregation module, AST-GRU further learns the long-term dependencies of the sequential features $\left\{\boldsymbol{X}_{t}\right\}_{t=1}^{T}$. In particular, at each time step $t$, the unit receives current input features $\boldsymbol{X}_{t}$ as well as the memory features $\boldsymbol{H}_{t-1}$, and produces the updated memory features $\boldsymbol{H}_{t}$ with an attentive gating mechanism. In this way, $\boldsymbol{H}_{t}$ preserves the information of both previous and current frames, which facilitates the subsequent object detection task. Finally, detection heads such as classification and regression network branches can be exploited on $\boldsymbol{H}_{t}$ to obtain the final detections $\left\{\boldsymbol{Y}_{t}\right\}_{t=1}^{T}$.

For training the $3 \mathrm{D}$ video object detector, both the anchorbased [3, 9] and anchor-free [5, 49] loss functions can be applied in our framework. Furthermore, our framework works in both the online and offline inference modes. In the online mode, only previous frames are employed to help the detection of the current frame. In the offline mode, both previous and 
later frames are viewed as supporting frames to produce detections in the current frame, which further enhances the detection performance.

\subsection{Message Passing Neural Network}

Message Passing Neural Network (MPNN) [45] is a general formulation, which unifies various promising neural networks that operate on graph-structured data [50, 41, 51, 42]. Formally, it defines a directed graph $\mathcal{G}=(\mathcal{V}, \mathcal{E})$, with node $v_{i} \in \mathcal{V}$ and edge $e_{i, j} \in \mathcal{E}$. The core of MPNN is to iteratively pass messages between different nodes and mine the diverse relations of them. At each time step $t$, let $\boldsymbol{h}_{i}^{t}$ denote the state features of node $v_{i}$, and $\boldsymbol{e}_{j, i}^{t}$ represents edge features of $e_{i, j}$ that describes the information passed from node $v_{j}$ to $v_{i}$. MPNN aggregates information for node $v_{i}$ with the neighbors $v_{j} \in \boldsymbol{\Omega}_{v_{i}}$, and infers its updated state features $\boldsymbol{h}_{i}^{t+1}$ based on the received messages. It runs for $K$ time steps and thus captures the long range interactions among the nodes.

More specifically, MPNN includes a message function $M(\cdot)$ and a node update function $U(\cdot)$. At each time step $t, M(\cdot)$ summarizes the message $\boldsymbol{m}_{j, i}^{t+1}$ passed from the neighbor node $v_{j} \in \boldsymbol{\Omega}_{v_{i}}$ to $v_{i}$, then obtains the aggregated message features $\boldsymbol{m}_{i}^{t+1}$. Notice that the message features $\boldsymbol{m}_{j, i}^{t+1}$ is computed by considering both the node state features and edge features, which is denoted as follows:

$$
\begin{aligned}
\boldsymbol{m}_{i}^{t+1} & =\sum_{j \in \boldsymbol{\Omega}_{i}} \boldsymbol{m}_{j, i}^{t+1} \\
& =\sum_{j \in \boldsymbol{\Omega}_{i}} M\left(\boldsymbol{h}_{i}^{t}, \boldsymbol{h}_{j}^{t}, \boldsymbol{e}_{j, i}^{t}\right) .
\end{aligned}
$$

Then, according to the collected message $\boldsymbol{m}_{i}^{t+1}$, the update functions $U(\cdot)$ refines the previous state features $\boldsymbol{h}_{i}^{t}$ for node $v_{i}$, and produces the updated state features $\boldsymbol{h}_{i}^{t+1}$ :

$$
\boldsymbol{h}_{i}^{t+1}=U\left(\boldsymbol{h}_{i}^{t}, \boldsymbol{m}_{i}^{t+1}\right) .
$$

In MPNN, both $M(\cdot)$ and $U(\cdot)$ are parameterized by weightsharing neural networks and all the operations can be learnt with gradient-based optimization. After one time step, a node accesses information from its neighbor nodes. After $K$ time step, the information from long-range nodes can be obtained. In this work, we extend MPNN to the context of LiDAR point clouds by treating grids as nodes. Our proposed GMPNet can encode the grid-wise features by mining the spatiotemporal relations in short-term point clouds.

\subsection{ConvGRU Network}

Gated Recurrent Unit (GRU) model [52] is a streamlined variant of Recurrent Neural Network (RNN) [53, 54, 55], which is originally devised for machine translation and video understanding by capturing the dependencies of input sequences. It simplifies the computation of RNN and achieves comparable performance. Later, Ballas et al. [10] proposed convolutional GRU (ConvGRU), which utilizes convolutional layers to replace the fully-connected ones in the original GRU. It not only preserves the better spatial resolution of the input features, but also largely reduces the number of parameters. ConvGRU has shown promising results on many vision tasks [7, 56, 57, 58]. Basically, ConvGRU contains an update gate $\boldsymbol{z}_{t}$, a reset gate $\boldsymbol{r}_{t}$, a candidate memory $\tilde{\boldsymbol{H}}_{t}$ and a current memory $\boldsymbol{H}_{t}$. At each time step, it computes the current memory $\boldsymbol{H}_{t}$ (or the hidden state) according to the previous memory $\boldsymbol{H}_{t-1}$ and the current input $\boldsymbol{X}_{t}$, which is denoted by the following equations:

$$
\begin{aligned}
\boldsymbol{z}_{t} & =\sigma\left(\boldsymbol{W}_{z} * \boldsymbol{X}_{t}+\boldsymbol{U}_{z} * \boldsymbol{H}_{t-1}\right), \\
\boldsymbol{r}_{t} & =\sigma\left(\boldsymbol{W}_{r} * \boldsymbol{X}_{t}+\boldsymbol{U}_{r} * \boldsymbol{H}_{t-1}\right), \\
\tilde{\boldsymbol{H}}_{t} & =\tanh \left(\boldsymbol{W} * \boldsymbol{X}_{t}+\boldsymbol{U} *\left(\boldsymbol{r}_{t} \circ \boldsymbol{H}_{t-1}\right)\right), \\
\boldsymbol{H}_{t} & =\left(\mathbf{1}-\boldsymbol{z}_{t}\right) \circ \boldsymbol{H}_{t-1}+\boldsymbol{z}_{t} \circ \tilde{\boldsymbol{H}}_{t},
\end{aligned}
$$

where '*' denotes the convolution operation, ' $\circ$ ' is the Hadamard product and $\sigma$ acts as a sigmoid activation function. $\boldsymbol{W}, \boldsymbol{W}_{z}, \boldsymbol{W}_{r}$ and $\boldsymbol{U}, \boldsymbol{U}_{z}, \boldsymbol{U}_{r}$ are the 2D convolutional kernels. The reset gate $\boldsymbol{r}_{t}$ determines how much of the past information from $\boldsymbol{H}_{t-1}$ to forget, so as to produce the candidate memory $\tilde{\boldsymbol{H}}_{t}$. For example, the information of $\tilde{\boldsymbol{H}}_{t}$ all comes from the current input $\boldsymbol{X}_{t}$ when $\boldsymbol{r}_{t}=0$. Besides, the update gate $\boldsymbol{z}_{t}$ decides the degree to which the current memory $\boldsymbol{H}_{t}$ accumulates the previous information form $\boldsymbol{H}_{t-1}$. Our ASTGRU significant improves the vanilla ConvGRU by integrating it with STA and TTA modules, which enforces the network to focus on meaningful objects in long-term point clouds.

\subsection{Grid Message Passing Network}

As introduced in $\$ 3.1$, in the short-term encoding module, we aggregate the $K$ short-term point cloud frames by concatenating them into a single frame. To extract features on this merged frame, dominant approaches tend to quantize the point clouds into regular grids such as voxels or pillars, and then utilize modules like Voxel Feature Encoding Layer [8] or the Pillar Feature Network [3] to encode the grids. These modules typically consider an individual grid, which fails to capture the spatiotemporal relations between the nodes from nearby frames, as well as limiting the expressive power due to the local representation. To this end, we propose Grid Message Passing Network (GPMNet) to mine the spatiotemporal relations of the grids, which results in a non-local representation. GMPNet views each non-empty grid as a node, and the relations between grids as edges. It iteratively passes messages between the grids and updates the grid-level representation accordingly. Besides, GPMNet also provides complementary perspectives for the subsequent $\mathrm{CNN}$ backbone with the nonEuclidean characteristics

Specifically, given a merged point cloud frame $\boldsymbol{I}_{t}$, we first uniformly discretize it into a set of grids $\mathcal{V}$. The grid can be either a voxel or a pillar, determined by the baseline detectors. Then a directed graph $\mathcal{G}=(\mathcal{V}, \mathcal{E})$ is constructed, where each node $v_{i} \in \mathcal{V}$ represents a non-empty grid and each edge $e_{j, i} \in \mathcal{E}$ holds the information passed from node $v_{j}$ to $v_{i}$. Furthermore, we define $\mathcal{G}$ as a $k$-nearest neighbor $(k$-NN) graph to save computations, which means that each node can directly access information from the $K$ spatial neighbors (also known as the first order neighbors). The goal of GMPNet is to adaptively update feature representation $\boldsymbol{h}_{i}$ for each grid-wise node $v_{i}$ by integrating information from long-range nodes, i.e., 
the higher order neighbors. Given a grid-wise node $v_{i}$, we first use a simplified PointNet [26] module to abstract its initial state features $\boldsymbol{h}_{i}^{0}$, which map a set of points within $v_{i}$ to an $L$-dim vector. The simplified PointNet is composed of fully connected layers $f(\cdot)$ and a max-pooling operation:

$$
\boldsymbol{h}_{i}^{0}=\max \left\{f\left(V_{i}\right)\right\} \in \mathbb{R}^{L},
$$

where $V_{i} \in \mathbb{R}^{N \times D}$ denotes the grid $v_{i}$ with $N$ point clouds parameterized by $D$-dim representation (eg., the XYZ coordinates and the reflectance of LiDAR).

Next, we elaborate the message passing and node state updating in GMPNet, following the formulation presented in $\$ 3.2$. At time step $s, v_{i}$ aggregates information from its neighbors $v_{j} \in \boldsymbol{\Omega}_{v_{i}}$ according to Eq. 1 . The incoming edge features $\boldsymbol{e}_{j, i}^{s}$ is defined as:

$$
\boldsymbol{e}_{j, i}^{s}=\boldsymbol{h}_{j}^{s}-\boldsymbol{h}_{i}^{s} \in \mathbb{R}^{L},
$$

which is an asymmetric function encoding the local neighbor information. Accordingly, the message passed from $v_{j}$ to $v_{i}$ is denoted as:

$$
\boldsymbol{m}_{j, i}^{s+1}=\phi_{\theta}\left(\left[\boldsymbol{h}_{i}^{s}, \boldsymbol{e}_{j, i}^{s}\right]\right) \in \mathbb{R}^{L^{\prime}},
$$

where $\phi_{\theta}$ is a fully connected layer, denoting the message function $M(\cdot)$ in Eq. 1. It receives the concatenation of $\boldsymbol{h}_{i}^{s}$ and $\boldsymbol{e}_{j, i}^{s}$, and yields an $L^{\prime}$-dim feature. We then summarize the received messages from $K$ neighbors with a max-pooling operation:

$$
\boldsymbol{m}_{i}^{s+1}=\max _{j \in \boldsymbol{\Omega}_{i}}\left\{\boldsymbol{m}_{j, i}^{s+1}\right\} \in \mathbb{R}^{L^{\prime}} .
$$

Afterwards, with the collected message $\boldsymbol{m}_{i}^{s+1}$, we update the state features $\boldsymbol{h}_{i}^{s}$ for node $v_{i}$ in terms of Eq. 2. Here, GRU [52] is used as the update function $U(\cdot)$ to adaptively preserve the information in different time steps, which is:

$$
\boldsymbol{h}_{i}^{s+1}=\operatorname{GRU}\left(\boldsymbol{h}_{i}^{s}, \boldsymbol{m}_{i}^{s+1}\right) \in \mathbb{R}^{L} .
$$

After one time step, $v_{i}$ contains the information from the neighbors $v_{j} \in \boldsymbol{\Omega}_{v_{i}}$. Moreover, the neighbor node $v_{j}$ also holds information from its own neighbors $\boldsymbol{\Omega}_{v_{j}}$. Therefore, $v_{i}$ can aggregate information from long-range nodes after a total of $S$ time steps. The message passing and node updating processes are illustrated in Fig. 3.

We obtain the final grid-wise feature representation $\boldsymbol{v}_{i}$ by applying another fully connected layer $\phi_{\theta}^{\prime}$ on $\boldsymbol{h}_{i}^{S}$ :

$$
\boldsymbol{v}_{i}=\phi_{\theta}^{\prime}\left(\boldsymbol{h}_{i}^{S}\right) \in \mathbb{R}^{L} .
$$

After that, all the grid-wise features are then scattered back to a regular tensor $\tilde{\boldsymbol{I}}_{t}$, e.g. , $\tilde{\boldsymbol{I}}_{t} \in \mathbb{R}^{W \times H \times L}$ with the PointPillars [3] baseline. Finally, we apply the CNN backbone in [8] to further extract features for $\tilde{\boldsymbol{I}}_{t}$ :

$$
\boldsymbol{X}_{t}=F_{\mathrm{B}}\left(\tilde{\boldsymbol{I}}_{t}\right) \in \mathbb{R}^{w \times h \times c},
$$

where $F_{\mathrm{B}}$ is the backbone network and $\boldsymbol{X}_{t}$ is the obtained short-term features of $\boldsymbol{I}_{t}$. This leads to a reduced resolution for $\boldsymbol{I}_{t}$, facilitating the subsequent long-term aggregation module. More details of the GMPNet and the CNN backbone can be found in $\S 4.2$.
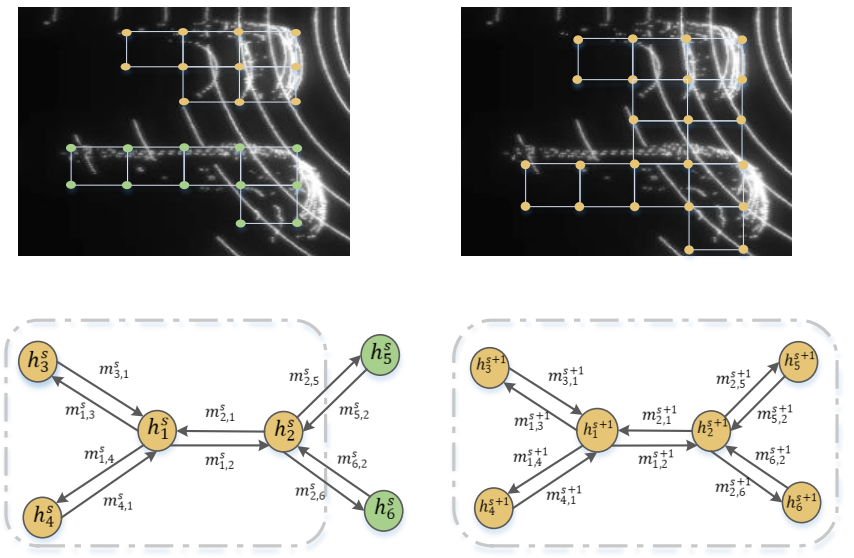

Fig. 3: Illustration of one iteration step for message propagation, where $h_{i}$ is the state of node $v_{i}$. In step $s$, the neighbors for $h_{1}$ are $\{h 2, h 3, h 4\}$ (within the gray dash line), presenting the grids of the top car. After receiving messages from the neighbors, the receptive field of $h_{1}$ is enlarged in step $s+1$. This indicates the relations with the bottom car are modeled after message propagation.

\subsection{Attentive Spatiotemporal Transformer GRU}

Recall that we have divided the input point cloud sequences $\left\{\boldsymbol{I}_{t}\right\}_{t=1}^{T \times M}$ into multiple short-term clips $\left\{\boldsymbol{I}_{t}\right\}_{t=1}^{T}$, with each clip $\boldsymbol{I}_{t}$ including concatenated point clouds from $M$ neighbor frames. Then, in the short-term encoding module, we independently described the short-term features $\boldsymbol{X}_{t}$ for each $\boldsymbol{I}_{t}$. Here, we further capture the long-term dynamics over the sequential features $\left\{\boldsymbol{X}_{t}\right\}_{t=1}^{T}$ in the long-term aggregation module by exploiting the Attentive Spatiotemporal Transformer GRU (AST-GRU). AST-GRU is an extension of ConvGRU that adaptively mines the spatiotemporal dependencies in $\left\{\boldsymbol{X}_{t}\right\}_{t=1}^{T}$. In particular, it improves the vanilla ConvGRU in two ways inspired by the transformer attention mechanism [11, 59]. First, dominant approaches usually perform detection on birdeye view feature maps $[3,5,8,9]$. However, the interest objects are much smaller in such a view compared with the front view in the 2D images. For example, using PointPillars [3] with a voxel size of $0.25^{2} \mathrm{~m}^{2}$, a vehicle (eg, with size of $4 m \times 2 m$ ) remains only 2 pixels in $\boldsymbol{X}_{t}$ after the feature extractor with common stride of 8 . This causes difficulties for the recurrent unit, because the background noise will inevitably accumulate across time in $\boldsymbol{H}_{t}$. To handle this problem, we propose a spatial transformer attention (STA) module by attending each pixel in $\boldsymbol{X}_{t}$ with its rich spatial context, which helps the detectors to focus on foreground objects. Second, for a recurrent unit, the received current input $\boldsymbol{X}_{t}$ and pervious memory $\boldsymbol{H}_{t-1}$ are not spatially aligned. Though we have aligned the static objects with the ego-pose information, the dynamic objects with large motion still lead to an inaccurate current memory $\boldsymbol{H}_{t}$. Therefore, a temporal transformer attention (TTA) module is introduced to align the moving objects in $\boldsymbol{H}_{t}$, which exploits modified deformable convolutional networks to capture the motion cues in $\boldsymbol{H}_{t-1}$ and $\boldsymbol{X}_{t}$. Next, we elaborate the workflow of our STA and TTA modules.

Spatial Transformer Attention. Motivated by the transformer 


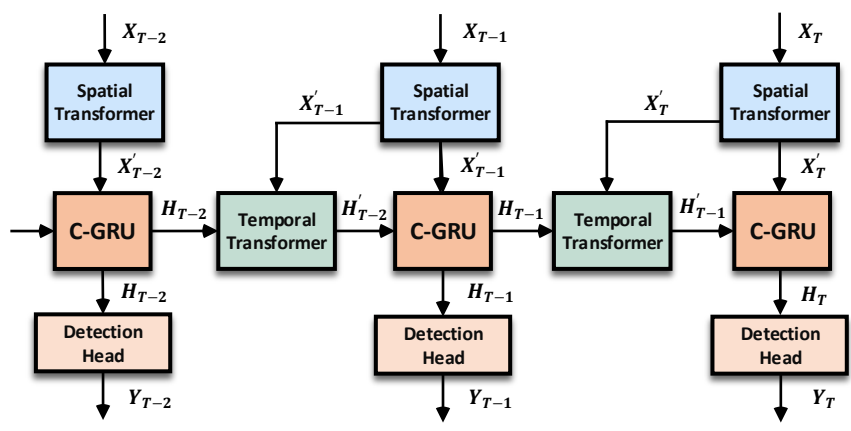

Fig. 4: Illustration of our proposed AST-GRU in the online mode. It consists of a spatial transformer attention (STA) module and a temporal transformer attention (TTA) module. AST-GRU captures the dependencies from a long-term perspective and produces the enhanced memory features $\left\{\boldsymbol{H}_{t}\right\}_{t=1}^{T}$.

attention in [11], we propose STA to stress the foreground pixels in $\boldsymbol{X}_{t}$ and suppress the background ones. In particular, each pixel $\boldsymbol{x}_{q} \in \boldsymbol{X}_{t}$ is considered as a query input and its context pixels $\boldsymbol{x}_{k} \in \boldsymbol{\Omega}_{\boldsymbol{x}_{q}}$ are viewed as keys. Since both the queries and keys are from the same feature map $\boldsymbol{X}_{t}$, STA can be grouped into an intra-attention family. For each query, its corresponding keys are embedded as values and the output of STA is the weighted sum of the values. We compute the weight of values by comparing the similarity between the query and the corresponding key, such that the keys that have the same class as the query could contribute to the output.

Formally, given an input query-key pair $\boldsymbol{x}_{q}, \boldsymbol{x}_{k} \in \boldsymbol{X}_{t}$, we first map them into different subspaces with linear layers $\phi_{Q}(\cdot), \phi_{K}(\cdot)$ and $\phi_{V}(\cdot)$ to get the embedded feature vectors for the query, key and value, respectively. Then, the attentive output $\boldsymbol{y}_{q}$ for a query $\boldsymbol{x}_{q}$ is calculated as:

$$
\boldsymbol{y}_{q}=\sum_{k \in \boldsymbol{\Omega}_{q}} A\left(\phi_{Q}\left(\boldsymbol{x}_{q}\right), \phi_{K}\left(\boldsymbol{x}_{k}\right)\right) \cdot \phi_{V}\left(\boldsymbol{x}_{k}\right),
$$

where $A(\cdot, \cdot)$ is the function to compute the attention weight. In practice, STA needs to compute the attention for all the query positions simultaneously. Therefore, we replace $\phi_{K}$, $\phi_{Q}$ and $\phi_{V}$ with the convolutional layers, $\Phi_{K}, \Phi_{Q}$ and $\Phi_{V}$, such that STA can be optimized with matrix multiplication operations. Specifically, the input features $\boldsymbol{X}_{t}$ are first embedded as $\boldsymbol{K}_{t}, \boldsymbol{Q}_{t}$ and $\boldsymbol{V}_{t} \in \mathbb{R}^{w \times h \times c^{\prime}}$ through $\Phi_{K}, \Phi_{Q}$ and $\Phi_{V}$. Then, we define the weight function $A(\cdot, \cdot)$ as a dotproduct operation followed by a softmax layer to measure the similarity of query-key pairs. To compute the attention weight $\tilde{\boldsymbol{A}}$, we reshape $\boldsymbol{K}_{t}$ and $\boldsymbol{Q}_{t}$ to $l \times c^{\prime}(l=w \times h)$ for saving computation:

$$
\tilde{\boldsymbol{A}}=\operatorname{softmax}\left(\boldsymbol{Q}_{t} \cdot \boldsymbol{K}_{t}^{T}\right) \in \mathbb{R}^{l \times l} .
$$

Afterwards, we multiply the attention weight $\tilde{\boldsymbol{A}}$ by values $\boldsymbol{V}_{t}$ to obtain the attention output $\tilde{\boldsymbol{A}} \cdot \boldsymbol{V}_{t}$. Next, the shape of the output is recovered back to $w \times h \times c^{\prime}$, and head layers $\boldsymbol{W}_{\text {out }}$ are employed to determine the specific mode of attention. Finally, we obtain the attention features $\boldsymbol{X}_{t}^{\prime}$ via a residual operation [60]. These steps can be summarized as:

$$
\boldsymbol{X}_{t}^{\prime}=\boldsymbol{W}_{\text {out }} *\left(\tilde{\boldsymbol{A}} \cdot \boldsymbol{V}_{t}\right)+\boldsymbol{X}_{t} \in \mathbb{R}^{w \times h \times c},
$$

where attention head $\boldsymbol{W}_{\text {out }}$ also maps the feature subspace of $\tilde{\boldsymbol{A}} \cdot \boldsymbol{V}_{t}\left(\right.$ e.g., $c^{\prime}$-dim) back to the original space (e.g., $c$-dim in $\boldsymbol{X}_{t}$ ). The resultant output $\boldsymbol{X}_{t}^{\prime}$ aggregates the information from the context pixels and thus can better focus on the small foreground objects, as well as suppressing the background noise accumulated in memory features.

Temporal Transformer Attention. The basic idea of TTA is to align the spatial features of $\boldsymbol{H}_{t-1}$ and $\boldsymbol{X}_{t}^{\prime}$, so as to give a more accurate current memory $\boldsymbol{H}_{t}$. Here, we utilize the modified deformable convolutional layers [13, 14] as a special instantiation of the transformer attention. TTA treats each pixel $\boldsymbol{h}_{q} \in \boldsymbol{H}_{t-1}$ as a query and determines the positions of keys by attending the current input $\boldsymbol{X}_{t}^{\prime}$, thus capturing the temporal motion information of moving objects. TTA belongs to the inter-attention since it involves both $\boldsymbol{H}_{t-1}$ and $\boldsymbol{X}_{t}^{\prime}$.

Specifically, we first describe the regular deformable convolutional network. Let $\boldsymbol{w}_{m}$ denote the convolutional kernel with size $3 \times 3$, and $p_{m} \in\{(-1,-1),(-1,0), \ldots,(1,1)\}$ indicate the predefined offsets of the kernel in $M=9$ grids. Given a pixel-wise input $\boldsymbol{h}_{q} \in \boldsymbol{H}_{t-1}$, the output $\boldsymbol{h}_{q}^{\prime}$ of this deformable convolutional layer can be expressed as:

$$
\boldsymbol{h}_{q}^{\prime}=\sum_{m=1}^{M} \boldsymbol{w}_{m} \cdot \boldsymbol{h}_{q+p_{m}+\Delta p_{m}},
$$

where $\Delta p_{m}$ is the deformation offset learnt through another regular convolutional layer $\Phi_{R}$ to model spatial transformations, i.e., $\Delta p_{m} \in \Delta \boldsymbol{P}_{t-1}=\Phi_{R}\left(\boldsymbol{H}_{t-1}\right) \in \mathbb{R}^{w \times h \times 2 r^{2}}$, where $2 r^{2}$ represnts the offsets in both $\mathrm{x}$ and $\mathrm{y}$ directions.

Following the perspective of transformer attention in Eq. 11, we could also reformulate Eq. 14 as:

$$
\boldsymbol{h}_{q}^{\prime}=\sum_{m=1}^{M} \sum_{k \in \boldsymbol{\Omega}_{q}}\left(\boldsymbol{w}_{m} \cdot G\left(k, q+p_{m}+\Delta p_{m}\right)\right) \cdot \phi_{\mathrm{V}}\left(\boldsymbol{h}_{k}\right),
$$

where $\boldsymbol{h}_{q}^{\prime}$ is the attentive output of the query $\boldsymbol{h}_{q}$ by a weighted sum on the $M=9$ values denoted by $\phi_{\mathrm{V}}\left(\boldsymbol{h}_{k}\right)$. Notice that $\phi_{\mathrm{V}}$ is an identity function here, such that $\phi_{\mathrm{V}}\left(\boldsymbol{h}_{k}\right)=\boldsymbol{h}_{k}$. The attention weight is calculated through the kernel wight $\boldsymbol{w}_{m}$ followed by a bilinear interpolation function $G(\cdot, \cdot)$ :

$$
G(a, b)=\max (0,1-|a-b|),
$$

which decides the sampling positions of the keys in the supporting regions $\boldsymbol{\Omega}_{q}$.

Since the interest objects have moved from $\boldsymbol{H}_{t-1}$ to $\boldsymbol{X}_{t}^{\prime}$, our TTA integrates the information in $\boldsymbol{X}_{t}^{\prime}$ to compute a refined offset $\Delta p_{m} \in \Delta \boldsymbol{P}_{t-1}$, and therefore adjust the sampling positions of the keys accordingly. In particular, we define a motion map with the difference of $\boldsymbol{H}_{t-1}$ and $\boldsymbol{X}_{t}^{\prime}$, and then compute $\Delta \boldsymbol{P}_{t-1}$ as:

$$
\Delta \boldsymbol{P}_{t-1}=\Phi_{R}\left(\left[\boldsymbol{H}_{t-1}, \boldsymbol{H}_{t-1}-\boldsymbol{X}_{t}^{\prime}\right]\right) \in \mathbb{R}^{w \times h \times 2 r^{2}},
$$

where $\Phi_{R}$ is a regular convolutional layer with the kernel size $3 \times 3$ to predict the offsets, and $[\cdot, \cdot]$ is the concatenation operation. The intuition of the motion map is that the features response of the static objects is very low since they have been spatially aligned in $\boldsymbol{H}_{t-1}$ and $\boldsymbol{X}_{t}^{\prime}$, while the features response of the moving objects remains high. By combining 
the salient features in the motion map, TTA could focus more on the moving objects. Afterwards, the output $\Delta \boldsymbol{P}_{t-1}$ is used to determine the regions of keys and further attend $\boldsymbol{H}_{t-1}$ for all the querys $q \in w \times h$ in terms of Eq. 15, yielding a temporally attentive memory $\boldsymbol{H}_{t-1}^{\prime}$. Additionally, we could stack multiple such modified deformable convolutional layers to further refine $\boldsymbol{H}_{t-1}^{\prime}$. In our implementation, two layers are employed, where the latter layer receives $\boldsymbol{H}_{t-1}^{\prime}$ and updates it similar to Eq.17.

Consequently, we have the temporally attentive previous memory $\boldsymbol{H}_{t-1}^{\prime}$ and the spatially attentive current input $\boldsymbol{X}_{t}^{\prime}$. This leads to an enhanced current memory $\boldsymbol{H}_{t}$ which contains richer spatiotemporal information and produces better detection results $\boldsymbol{Y}_{t}$ after being equipped with detection heads (see Fig. 4). We have described our AST-GRU with the online detection setting. In $\S 3.6$, we present the offline detection setting by exploiting bidirectional AST-GRU.

\subsection{Offline 3D Video Object Detection}

With the proposed AST-GRU, we have achieved better performance for online 3D video object detection, which is the common case in autonomous driving. However, we could provide a stronger 3D video object detector by exploring both the past and future frames, which will facilitate more casual applications. For example, when annotating LiDAR frames for autonomous driving scenes, it is easy to access future frames in a scene. Our offline 3D video object detector could act as an annotation tool by providing more accurate initial 3D bounding boxes. This may greatly increase the productivity of annotators compared with using a frame-by-frame detector. To this end, we further adapt AST-GRU to the bidirectional offline setting by capturing temporal information in both past and future frames.

Concretely, by considering the attentive current input $\boldsymbol{X}_{t}^{\prime}$ and the previous memory $\boldsymbol{H}_{t-1}^{\prime}$, we have acquired the forward memory features $\boldsymbol{H}_{t}^{f}$. To get a more powerful representation for the current frame, we could better integrate the backward information from future features $\boldsymbol{H}_{t+1}^{\prime}$, Similar to Eq. 3, we use the following equations to capture the rich temporal information preserved in $\boldsymbol{H}_{t+1}^{\prime}$ and compute the backward memory features $\boldsymbol{H}_{t}^{b}$ :

$$
\begin{aligned}
& \boldsymbol{z}_{t}^{b}=\sigma\left(\boldsymbol{W}_{z}^{b} * \boldsymbol{X}_{t}^{\prime}+\boldsymbol{U}_{z}^{b} * \boldsymbol{H}_{t+1}^{\prime}\right), \\
& \boldsymbol{r}_{t}^{b}=\sigma\left(\boldsymbol{W}_{r}^{b} * \boldsymbol{X}_{t}^{\prime}+\boldsymbol{U}_{r}^{b} * \boldsymbol{H}_{t+1}^{\prime}\right), \\
& \tilde{\boldsymbol{H}}_{t}^{b}=\tanh \left(\boldsymbol{W}^{b} * \boldsymbol{X}_{t}^{\prime}+\boldsymbol{U}^{b} *\left(\boldsymbol{r}_{t}^{b} \circ \boldsymbol{H}_{t+1}^{\prime}\right)\right), \\
& \boldsymbol{H}_{t}^{b}=\left(\mathbf{1}-\boldsymbol{z}_{t}^{b}\right) \circ \boldsymbol{H}_{t+1}^{\prime}+\boldsymbol{z}_{t}^{b} \circ \tilde{\boldsymbol{H}}_{t}^{b},
\end{aligned}
$$

where $\boldsymbol{W}^{b}, \boldsymbol{W}_{z}^{b}, \boldsymbol{W}_{r}^{b}$ and $\boldsymbol{U}^{b}, \boldsymbol{U}_{z}^{b}, \boldsymbol{U}_{r}^{b}$ are sets of learnable parameters included in the backward AST-GRU. The reset gate $\boldsymbol{r}_{t}^{b}$ and update gate $\boldsymbol{z}_{t}^{b}$ measure the importance of the future information $\boldsymbol{H}_{t+1}^{\prime}$ to the current input $\boldsymbol{X}_{t}^{\prime}$.

In this way, our bidirectional AST-GRU obtains the memory features from both forward and backward units. Then, we combine these features with a concatenation operation to aggregate the information and get an enhanced memory $\boldsymbol{H}_{t}^{\prime}$, which is calculated as:

$$
\boldsymbol{H}_{t}^{\prime}=\left[\boldsymbol{H}_{t}^{f}, \boldsymbol{H}_{t}^{b}\right] \in \mathbb{R}^{w \times h \times 2 c} .
$$

Afterwards, detection heads can be directly applied on $\boldsymbol{H}_{t}^{\prime}$ to produce the 3D detection results. The proposed bidirectional AST-GRU exploits the spatiotemporal dependencies from both past and future, which offers complementary cues for detecting in the current frame. In $\S 4.4$, we will prove that it outperforms the unidirectional AST-GRU by a large margin.

\section{Experimental Results}

We empirically evaluate our algorithm on the large-scale nuScenes benchmark that will be introduced $\S 4.1$. Since our framework is agnostic to the detectors, we demonstrate the performance of our algorithm based on two prevalent 3D object detectors: anchor-based PointPillars [3] and anchor-free CenterPoint [5]. The detailed network architecture and main detection results are presented in $\S 4.2$ and $\S 4.3$, respectively. Afterwards, we provide thorough and comprehensive ablation studies to assess each module of our algorithm in $\S 4.4$.

\subsection{D Video Object Detection Benchmark}

Since the point cloud videos are not available in the commonly used KITTI benchmark [71], we turn to the more challenging nuScenes benchmark [2] to evaluate our algorithm and compare with other approaches on the leaderboard. The nuScenes dataset is composed of 1,000 driving scenes (i.e., video clips), with each scene containing around 400 frames of 20-second in length. The annotations are conducted every 10 frames for 10 object classes, and the training set provides $7 \times$ as many annotations as the KITTI dataset. There are 700 scenes $(28,130$ annotations) for training, 150 scenes $(6,019$ annotations) for validation and 150 scenes (6,008 annotations) for testing. Besides, nuScenes utilizes a 32-beam LiDAR with $20 \mathrm{~Hz}$ capture frequency, resulting in approximately $100 \mathrm{k}$ points in a full 360-degree view. The official evaluation protocol for 3D object detection defines an NDS (nuScenes detection score) metric, which is a weighted sum of mean Average Precision (mAP) and several True Positive (TP) metrics. Different from the definition in KITTI, the mAP in nuScenes measures the varying center distance (i.e., $0.5 m, 1 m, 2 m$ and $4 m$ ) between the predictions and ground truths in the bird's eye view. Other TP metrics consider multiple aspects to measure the quality of the predictions, i.e., box location, size, orientation, attributes and velocity.

To build our point cloud-based video object detector, we follow the common implementation in nuScenes benchmark by merging the 10 previous LiDAR sweeps $(0.5 s)$ to corresponding keyframes with the ego-pose information. These LiDAR sweeps are deemed as short-term data, while the merged keyframes are viewed as long-term data. For our online model, two previous keyframes $(1 s)$ are used to detect in the current frame. As for the offline model, we use $0.5 s$ temporal information from both previous and future keyframes.

\subsection{Implementation Details}

Architecture. We achieve 3D video object detection based on both anchor-based and anchor-free LiDAR-based detectors. 


\begin{tabular}{|c|c|c|c|c|c|c|c|c|c|c|c|c|c|}
\hline Method & Publication & NDS & $\mathbf{m A P}$ & Car & Truck & Bus & Trailer & $\mathbf{C V}$ & Ped & Motor & Bicycle & TC & Barrier \\
\hline InfoFocus [61] & ECCV 2020 & 39.5 & 39.5 & 77.9 & 31.4 & 44.8 & 37.3 & 10.7 & 63.4 & 29.0 & 6.1 & 46.5 & 47.8 \\
\hline PointPillars [3] & CVPR 2019 & 45.3 & 30.5 & 68.4 & 23.0 & 28.2 & 23.4 & 4.1 & 59.7 & 27.4 & 1.1 & 30.8 & 38.9 \\
\hline WYSIWYG [4] & CVPR 2020 & 41.9 & 35.0 & 79.1 & 30.4 & 46.6 & 40.1 & 7.1 & 65.0 & 18.2 & 0.1 & 28.8 & 34.7 \\
\hline SARPNET [62] & NEURCO. 2020 & 48.4 & 32.4 & 59.9 & 18.7 & 19.4 & 18.0 & 11.6 & 69.4 & 29.8 & 14.2 & 44.6 & 38.3 \\
\hline 3DSSD [63] & CVPR 2020 & 56.4 & 42.6 & 81.2 & 47.2 & 61.4 & 30.5 & 12.6 & 70.2 & 36.0 & 8.6 & 31.1 & 47.9 \\
\hline PointPainting [64] & CVPR 2020 & 58.1 & 46.4 & 77.9 & 35.8 & 36.2 & 37.3 & 15.8 & 73.3 & 41.5 & 24.1 & 62.4 & 60.2 \\
\hline ReconfigPP [65] & ARXIV 2020 & 59.0 & 48.5 & 81.4 & 38.9 & 43.0 & 47.0 & 15.3 & 72.4 & 44.9 & 22.6 & 58.3 & 61.4 \\
\hline PointPillars_DSA [66] & ARXIV 2020 & 59.2 & 47.0 & 81.2 & 43.8 & 57.2 & 47.8 & 11.3 & 73.3 & 32.1 & 7.9 & 60.6 & 55.3 \\
\hline SSN V2 [6] $]$ & ARXIV 2020 & 61.6 & 50.6 & 82.4 & 41.8 & 46.1 & 48.0 & 17.5 & 75.6 & 48.9 & 24.6 & 60.1 & 61.2 \\
\hline $3 \mathrm{DCVF}$ [29] & ECCV 2020 & 62.3 & 52.7 & 83.0 & 45.0 & 48.8 & 49.6 & 15.9 & 74.2 & 51.2 & 30.4 & 62.9 & 65.9 \\
\hline CBGS [68] & ARXIV 2019 & 63.3 & 52.8 & 81.1 & 48.5 & 54.9 & 42.9 & 10.5 & 80.1 & 51.5 & 22.3 & 70.9 & 65.7 \\
\hline CVCNet [69] & NeurIPS 2020 & 64.2 & 55.8 & 82.7 & 46.1 & 45.8 & 46.7 & 20.7 & 81.0 & 61.3 & 34.3 & 69.7 & 69.9 \\
\hline HotSpotNet [49] & ECCV 2020 & 66.0 & 59.3 & 83.1 & 50.9 & 56.4 & 53.3 & 23.0 & 81.3 & 63.5 & 36.6 & 73.0 & 71.6 \\
\hline CyliNet [70] & ARXIV 2020 & 66.1 & 58.5 & 85.0 & 50.2 & 56.9 & 52.6 & 19.1 & 84.3 & 58.6 & 29.8 & 79.1 & 69.0 \\
\hline CenterPoint [5] & CVPR 2021 & 67.3 & 60.3 & 85.2 & 53.5 & 63.6 & 56.0 & 20.0 & 84.6 & 59.5 & 30.7 & 78.4 & 71.1 \\
\hline PointPillars-VID (Ours) & CVPR 2020 & 53.1 & 45.4 & 79.7 & 33.6 & 47.1 & 43.1 & 18.1 & 76.5 & 40.7 & 7.9 & 58.8 & 48.8 \\
\hline CenterPoint-VID (Ours) & - & 71.4 & 65.4 & 87.5 & 56.9 & 63.5 & 60.2 & 32.1 & 82.1 & 74.6 & 45.9 & 78.8 & 69.3 \\
\hline CenterPoint-VID* (Ours) & - & 71.8 & 67.4 & 87.0 & 58.0 & 67.1 & 60.2 & 31.0 & 88.2 & 76.5 & 51.2 & 85.2 & 69.7 \\
\hline
\end{tabular}

TABLE 1: Quantitative detection results on the nuScenes 3D object detection benchmark. T.C. presents the traffic cone. Moto. and Cons. are short for the motorcycle and construction vehicle, respectively. Our 3D video object detector significantly improves the single-frame detectors, and outperforms all the competitors on the leaderboard.

For the anchor-based baseline, we choose the official PointPillars [3] provided by nuScene benchmark. For the anchorfree baseline, the CenterPoint model [5] with VoxelNet [8] encoder is adopted in our framework. Specifically, for each merged point cloud keyframe, we define 5-dim input features $(x, y, z, r, \Delta t)$ for the points, where $r$ is the LiDAR intensity and $\Delta t$ formulates the time lag to the keyframe that ranges from $0 s$ to $0.5 s$. We consider the points whose coordinates locate within $[-61.2,61.2] \times[-61.2,61.2] \times[-10,10]$ meters along the $\mathrm{X}, \mathrm{Y}$ and $\mathrm{Z}$ axes. The grid size for grouping points is set as $0.25 \times 0.25 \times 8$ in PointPillars. While in the CenterPoint, we adopt a smaller size, $0.075 \times 0.075 \times 0.2$, to obtain better detection performance and compare with other approaches on the leaderboard. In the subsequent content, we mainly elaborate the architecture details based on the PointPillars due to space limitations, and all the modifications can be applied on CenterPoint accordingly.

In the short-term encoding module, we implement GMPNet by sampling 16,384 grid-wise nodes with Farthest Point Sampling algorithm [26], and build the $k$-NN graph with $K=20$ neighbors. A grid $v_{i}$ contains $N=60$ points with $D=5$ representations, which is embedded into feature space with channel number $L=64$ to get the node features. This is achieved by a $1 \times 1$ convolutional layer followed by a maxpooling operation, which yields the initial state features $G^{0} \in$ $\mathbb{R}^{16,384 \times 64}$ (Eq. 4) for all the nodes. Then, in the iteration step $s$, we obtain the message features $M^{s} \in \mathbb{R}^{16,384 \times 64}$ by performing $1 \times 1$ convolutional layer and max-pooling over the neighbors based on the edge features $E^{s} \in \mathbb{R}^{16,384 \times 20 \times 128}$ (Eq. 5 to Eq. 7.). Afterwards, the updated node state $G^{s+1} \in$ $\mathbb{R}^{16,384 \times 64}$ is computed by applying GRU on $G^{s}$ and $M^{s}$ with linear layers ((Eq. 8). After $S=3$ iteration steps, GMP produces the final node state $G^{3} \in \mathbb{R}^{16,384 \times 64}$ according to Eq. 9, and broadcast it to the bird's eye view. Next, we apply Region Proposal Network (RPN) [8] as the 2D backbone to further extract the features in the bird's eye view. RPN is composed of several convolutional blocks, with each defined as a tuple $(S, Z, C) . S$ represents the stride of each block, while $Z$ denotes the kernel size of each convolutional layer and $C$ is the output channel number. The output features of each block are resized to the same resolution via upsampling layers and concatenated together, so as to merge the semantic information from different levels.

In the long-term aggregation module, we implement the embedding functions $\Phi_{K}, \Phi_{Q}, \Phi_{V}$ and the output layer $\boldsymbol{W}_{\text {out }}$ in STA with $1 \times 1$ convolutional layers, and all the channel number is half of the inputs except $\boldsymbol{W}_{\text {out }}$ to save computations. In the TTA module, the regular convolutional layers, the deformable convolutional layers and the ConvGRU all have learnable kernels of size $3 \times 3$. Besides, all these convolutional kernels use the same channel number with the input bird's eye view features. We follow the PointPillars [3] baseline to set the anchor-based detection head and calculate anchors for different classes using the mean sizes. Two separate convolutional layers are used for classification and regression, respectively. For the anchor-free head, we refer to the CenterPoint baseline [5] by applying a center heatmap head and an attribute regression head. The former head aims to predict the center location of objects, while the latter head estimates a sub-voxel center location, as well as the box height, size, rotation and velocity.

Training and Inference. We train our point cloud-based video object detector following the pre-training and finetuning paradigm. Specifically, the short-term encoding module is first trained in the same way as a single-frame detector, where one-cycle learning rate policy is used for 20 epochs with a maximum learning rate of 0.003 for PointPillars and 0.001 for CenterPoint. Then, we fine-tune the whole video detector including the long-term aggregation module with a fixed learning rate $(0.003$ for PointPillars or 0.001 for CenterPoint) for 10 epochs. Corresponding anchor-based or anchor-free loss functions are utilized in each of the longterm keyframes. Adam optimizer [72] is used to optimize the loss functions in both stages. In our framework, we feed at most 3 keyframes (with 30 LiDAR sweeps) to the model due to memory limitation. At the inference time, we preserve at 
most 500 detections after Non-Maxima Suppression (NMS) with a score threshold of 0.1 . We test our algorithm on a Tesla v100 GPU and get an average speed of 5 FPS and 1 FPS for PointPillars and CenterPoint baselines, respectively.

\subsection{Quantitative and Qualitative Performance}

We validate the performance of our 3D video object detection algorithm on the test set of nuScenes benchmark by comparing it with other state-of-the-art works on the leaderboard. As shown in Table 1, our best model, i.e., with CenterPoint baseline, outperforms all the published methods in terms of nuScenes detection score (NDS), which is the most important metric in nuScenes. Furthermore, we achieve 1st on the leaderboard by the time the paper is submitted, without any tricks like ensemble modeling or leveraging information from 2D images.

Specifically, our anchor-based video detection model (PointPillars-VID) improves the PointPillars [3] baseline by nearly $8 \%$ NDS and 15\% mAP, respectively. This demonstrates the significance of integrating temporal information in 3D point clouds. The PointPillars is lightweight compared with other state-of-the-art methods. To further validate the performance of our algorithm, we implement it based on the stronger CenterPoint [5] baseline. CenterPoint proposes to represent objects as points with an anchor-free detection head and addresses the class imbalance problem inspired by CBGS [68], thus obtaining much better results. The newly released version of CenterPoint includes a two-stage refinement pipeline, while we only use the one-stage version as the baseline. By adopting the video detection strategy, our model (CenterPoint-VID) with offline mode advances a better performance, achieving 71.4\% NDS and $65.4 \mathrm{mAP}$ on the leaderboard. This significantly improves the strong baseline by $4.1 \% \mathrm{NDS}$ and $5.1 \% \mathrm{mAP}$. By further integrating the painting strategy proposed by PointPainting [64], our final model (CenterPoint-VID*) gives the best performance on the leaderboard i.e., achieving 71.8\% NDS and $67.4 \mathrm{mAP}$, without any sophisticated ensemble strategies.

In addition to the quantitative results in Table 1, we further provide some qualitative examples. We mainly compare our 3D video object detector with the single-frame detector baseline, i.e., PointPillars [3]. We show specific cases that our model outperforms the single-frame detector, e.g., objects are occluded in certain frames or there are distant or small objects with sparse points. Here, three consecutive frames are shown for each case. The occlusion case is presented in Fig. 1, where our video object detector can handle the occluded objects with the memory features in previous frames. In Fig. 5(a), we showcase the detection of the distant car (the car on the top right), whose point clouds are especially sparse. Though it is very challenging for the single-frame detectors, our 3D video object detector still improves the detection results thanks to the information from adjacent frames. Similar improvement is observed In Fig. 5(b), where a single-frame detector fails to detect the small objects in the right of the ego-car, while our model accurately recognizes these small objects. In a nutshell, compared with the single-frame detector, much fewer false

\begin{tabular}{c|c|cc}
\hline \multirow{2}{*}{ Components } & Modules & \multicolumn{2}{|c}{ Performance } \\
& mAP & $\Delta$ \\
\hline Short-term Point & Concatenation [3] & 21.30 & - \\
Cloud Encoding & GMPNet & 23.35 & +2.05 \\
\hline \multirow{4}{*}{ Long-term } & 3D ConvNet [6] & 23.12 & +1.82 \\
Point Cloud & ConvGRU [10] & 23.83 & +2.53 \\
Aggregation & STA-GRU & 25.23 & +3.93 \\
& TTA-GRU & 25.32 & +4.02 \\
& AST-GRU & 27.28 & +5.98 \\
\cline { 2 - 4 } & Full Model (online) & 29.35 & +8.05 \\
& Full Model (offline) & $\mathbf{3 0 . 8 2}$ & $\mathbf{+ 9 . 5 2}$ \\
\hline
\end{tabular}

TABLE 2: Ablation study of our 3D video object detector. PointPillars with concatenated point clouds [3] is the reference baseline for computing the relative improvement $(\Delta)$.

positive (FP) and false negative (FN) detection results are obtained in our video object detector.

\subsection{Ablation Studies}

In this section, we conduct ablation studies over the full validation set to verify each module of our algorithm. Since the nuScenes provides a larger training set that is $7 \times$ as large as that in the KITTI dataset ( 28,130 vs 3,712$)$, it is non-trivial to train on the whole training set with our video detector. To perform the diagnostic experiments, we adopt a smaller training set containing 3,517 frames, which is obtained by uniformly downsampling the original training set for 8 times. Besides, we utilize PointPillars [3] with concatenated point clouds as the baseline video detector for this paradigm is widely used in practice.

Comparison with other temporal encoders. Our algorithm benefits from both the short-term and long-term point cloud sequence information, where GMPNet and AST-GRU modules are devised to handle these two different temporal pattern$\mathrm{s}$, respectively. Here, we compare our modules with other temporal-based design strategies. In particular, for encoding the short-term point cloud features, the concatenation baseline [3] trained with merged point clouds, can be deemed as the simplest temporal encoder. As shown in Table 2, our GMPNet improves this strategy by $2.05 \% \mathrm{mAP}$. This demonstrates that GMPNet could effectively mine the motion features in short-term point clouds by exchanging messages among grids from nearby frames, while the baseline encoder, e.g., the Pillar Feature Network, only considers each grid independently. Furthermore, to verify the effectiveness of aggregating long-term point clouds, we compare our AST-GRU with 3D temporal ConvNet [6] and vanilla ConvGRU [10] methods. Since the 3D ConvNet could only enforce optimization on a single keyframe, it thus achieves worse results than the ConvGRU [10]. Though the ConvGRU has exploited the multiframe features, it ignores the influence of noisy background and the misalignment in spatial features. By contrast, our proposed AST-GRU module improves these two approaches by $4.16 \% \mathrm{mAP}$ and $3.46 \% \mathrm{mAP}$ respectively, according to Table 2. Besides, all the designs in AST-GRU give better performance. For example, the STA module enhances the ConvGRU by $1.40 \%$ mAP, while the TTA module further advances the performance by $2.05 \%$. The overall model containing both 
1

2

3

4

5

6

7

8

9
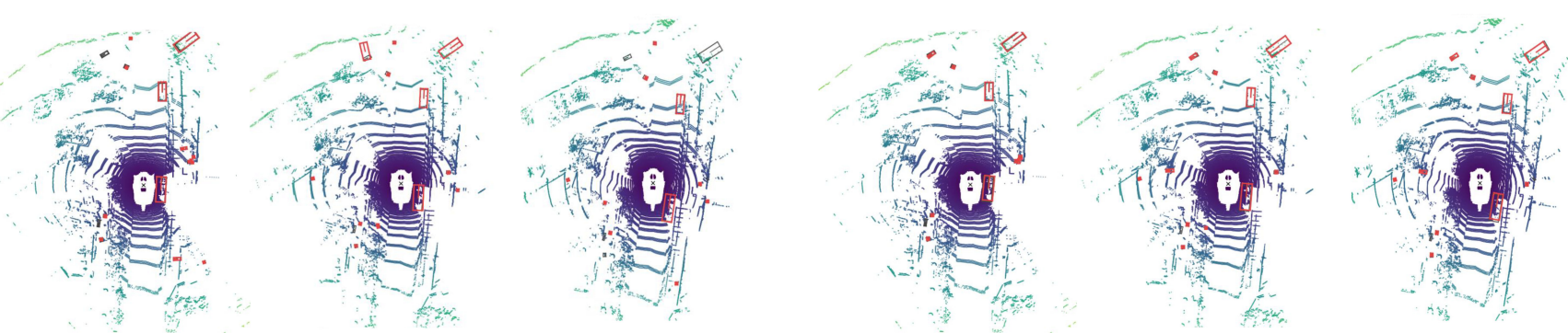

(a) The single-frame detector (left) fail to detect the car on the top right in the third frame, while our method (right) could address this.
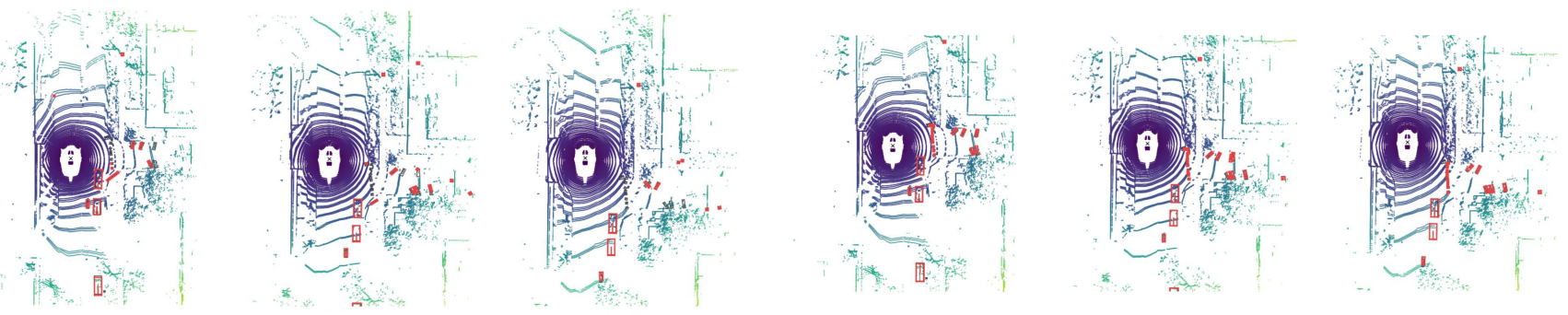

(b) The single-frame detector (left) have difficulty in detecting the small objects in the right of the ego-car (zoom in for better view).

Fig. 5: Qualitative results of 3D video object detection. We compare our algorithm (the right three frames in each case) with the single-frame 3D object detector (the left ones). The red and grey boxes indicate the predictions and ground-truths, respectively.

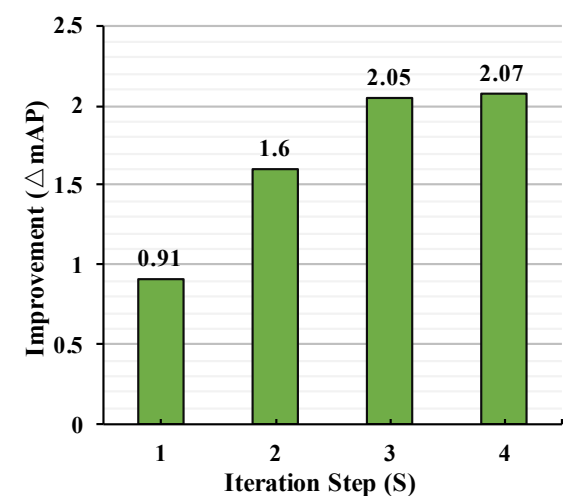

(a) Performance affected by $S(K=20)$.

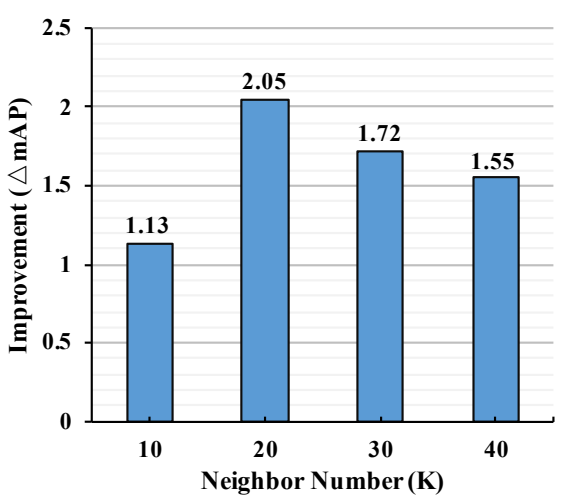

(b) Performance affected by $K(S=3)$.

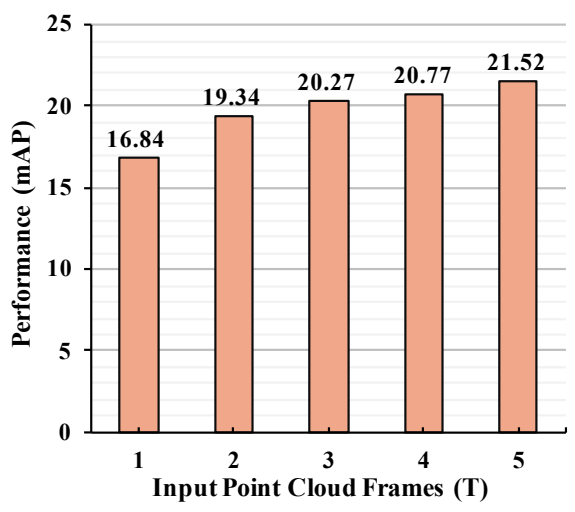

(c) Performance affected by $T$.

Fig. 6: Ablation study for GMPNet and the input length. In (a) and (b), we fix one parameter and vary the other to ablate the importance of iteration step $S$ and neighbor node number $K$. In (c), different keyframes are used to assess the effectiveness of our algorithm.

GMPNet and AST-GRU surpasses the baseline by $8.05 \% \mathrm{mAP}$. Moreover, we obtain the best model by integrating the offline strategy, further improving the online model by $1.47 \%$. This shows that the information from future frames can further boost the detection performance. Next, we ablate some crucial designs in GMPNet and TTA, as well as the influence of the length of input point cloud sequences.

Hyperparameters in GMPNet and Input length. Our GMPNet enables a grid to capture a flexible receptive field via iteratively propagating message on a $k$-NN graph. Given a grid-wise node, both the number of first-order neighbors (denoted as $\mathrm{K}$ ) and the total iteration steps (denoted as S) have an influence on the receptive field as well as the final performance. In order to clearly demonstrate the impact of these two parameters, we show the performance change by

\begin{tabular}{c|c|c|c}
\hline \multirow{2}{*}{ Aspect } & \multirow{2}{*}{ Modules } & \multicolumn{2}{|c}{ Performance } \\
& & mAP & $\Delta$ \\
\hline \multirow{3}{*}{$\begin{array}{c}\text { Inputs } \\
(\mathrm{M}=2)\end{array}$} & Full Model & $\mathbf{2 7 . 2 8}$ & $\mathbf{0}$ \\
\cline { 2 - 4 } & w/o motion map & 26.03 & -1.25 \\
\cline { 2 - 4 } w/ current input & 26.27 & -1.01 \\
\hline \multirow{2}{*}{$\begin{array}{c}\text { Layer } \\
\text { Number }\end{array}$} & $\mathbf{M}=1$ & 26.55 & -0.73 \\
\cline { 2 - 4 } & $\mathbf{M = 2}$ & $\mathbf{2 7 . 2 8}$ & $\mathbf{0}$ \\
\cline { 2 - 4 } & $\mathbf{M}=3$ & 26.42 & -0.86 \\
\hline
\end{tabular}

TABLE 3: Detailed analysis of the input choices and the layer number in TTA module. The full model is viewed as the reference for computing the relative performance $(\Delta)$.

varying $\mathrm{S}$ and $\mathrm{K}$. According to Fig. 6(a), we observe that $\mathrm{S}=3$ has already obtained enough receptive field, and further 
increasing $\mathrm{S}$ does not help promote the results. In Fig. 6(b), we show that $\mathrm{K}=20$ achieves the best performance, while a larger $\mathrm{K}$ instead degrades the performance. We infer that $\mathrm{K}=20$ and $\mathrm{S}=3$ have captured an appropriate receptive field, and a much larger receptive field may confuse the detector.

Different design strategies in TTA. Our TTA aligns the features of the dynamic objects by applying a modified deformable convolutional network. Thus, there are several factors affecting the final results, e.g., the inputs for computing supporting regions and the layer number of TTA. In our implementation, we integrate a motion map into the inputs and use two layers for TTA. Here, we give a detailed analysis of these aspects. As shown in Table. 3, w/o motion map denotes that TTA takes only the previous memory feature $\mathrm{H}_{t-1}$ as input, while w/ current input represents that TTA receives the concatenation of $\mathrm{H}_{t-1}$ and $\mathrm{X}_{t}^{\prime}$. Both designs give the decreased performance, demonstrating the effectiveness of the motion map. In addition, the model using two modified deformable convolutional layers achieves the best performance. We infer that the deeper layers lead to difficulty in optimizing the whole network.

Input length of point cloud frames. Finally, we analyze the effect of the input sequence length. Since each keyframe contains 10 LiDAR sweeps that significantly increase the memory demand, we conduct this experiment in the online mode without using the LiDAR sweeps (i.e., using only the long-term data). According to the results in Fig. 6(c), we observe that longer temporal information could consistently gain better performance in our framework.

\section{Conclusions}

In this paper, we presented a novel framework for 3D video object detection in point clouds. Our framework formulates the temporal information with short-term and long-term patterns, and devises a short-term encoding module and a long-term aggregation module to address these two temporal patterns. In the former module, a Grid Message Passing Network (GMPNet) is introduced to mine the short-term object motion cues in nearby point cloud frames. This is achieved by iterative message exchanging in a $k$-NN graph, e.g., a grid update its feature by integrating information from $k$ neighbor grids. In the latter module, an Attentive Spatiotemporal Transformer GRU (AST-GRU) is proposed to further aggregate long-term features. AST-GRU includes a Spatial Transformer Attention (STA) and a Temporal Transformer Attention (TTA), which are designed to handle small objects and align moving objects, respectively. Our point cloud video-based framework could work in both online and offline mode, depending on the applications. We also tested our framework with both anchor-based and anchor-free 3D object detectors. Evaluation results on the nuScenes benchmark demonstrate the superior performance of our framework.

\section{RefEREnCES}

[1] J. Yin, J. Shen, C. Guan, D. Zhou, and R. Yang, "Lidar-based online $3 \mathrm{~d}$ video object detection with graph-based message passing and spatiotemporal transformer attention," in Proceedings of the
IEEE Conference on Computer Vision and Pattern Recognition, pp. 11495-11504, 2020.

[2] H. Caesar, V. Bankiti, A. H. Lang, S. Vora, V. E. Liong, Q. Xu, A. Krishnan, Y. Pan, G. Baldan, and O. Beijbom, "nuscenes: A multimodal dataset for autonomous driving," in Proceedings of the IEEE Conference on Computer Vision and Pattern Recognition, pp. 11618-11628, 2020.

[3] A. H. Lang, S. Vora, H. Caesar, L. Zhou, J. Yang, and O. Beijbom, "Pointpillars: Fast encoders for object detection from point clouds," in Proceedings of the IEEE Conference on Computer Vision and Pattern Recognition, pp. 12697-12705, 2019.

[4] P. Hu, J. Ziglar, D. Held, and D. Ramanan, "What you see is what you get: Exploiting visibility for 3d object detection," in Proceedings of the IEEE Conference on Computer Vision and Pattern Recognition, pp. 10998-11006, 2020.

[5] T. Yin, X. Zhou, and P. Krähenbühl, "Center-based 3d object detection and tracking," arXiv preprint arXiv:2006.11275, 2020.

[6] W. Luo, B. Yang, and R. Urtasun, "Fast and furious: Real time end-to-end $3 \mathrm{~d}$ detection, tracking and motion forecasting with a single convolutional net," in Proceedings of the IEEE Conference on Computer Vision and Pattern Recognition, pp. 3569-3577, 2018.

[7] D. Tran, H. Wang, L. Torresani, J. Ray, Y. LeCun, and M. Paluri, "A closer look at spatiotemporal convolutions for action recognition," in Proceedings of the IEEE Conference on Computer Vision and Pattern Recognition, pp. 6450-6459, 2018.

[8] Y. Zhou and O. Tuzel, "Voxelnet: End-to-end learning for point cloud based 3d object detection," in Proceedings of the IEEE Conference on Computer Vision and Pattern Recognition, pp. 4490-4499, 2018.

[9] Y. Yan, Y. Mao, and B. Li, "Second: Sparsely embedded convolutional detection," Sensors, 18(10):3337, 2018.

[10] N. Ballas, L. Yao, C. Pal, and A. Courville, "Delving deeper into convolutional networks for learning video representations," in International Conference on Learning Representations, 2016.

[11] A. Vaswani, N. Shazeer, N. Parmar, J. Uszkoreit, L. Jones, A. N. Gomez, Ł. Kaiser, and I. Polosukhin, "Attention is all you need," in Advances in Neural Information Processing Systems, pp. 59986008, 2017.

[12] X. Wang, R. Girshick, A. Gupta, and K. He, "Non-local neural networks," in Proceedings of the IEEE Conference on Computer Vision and Pattern Recognition, pp. 7794-7803, 2018.

[13] X. Zhu, H. Hu, S. Lin, and J. Dai, "Deformable convnets v2: More deformable, better results," in Proceedings of the IEEE Conference on Computer Vision and Pattern Recognition, pp. 9308-9316, 2019.

[14] X. Zhu, D. Cheng, Z. Zhang, S. Lin, and J. Dai, "An empirical study of spatial attention mechanisms in deep networks," in Proceedings of the IEEE International Conference on Computer Vision, pp. 6688-6697, 2019.

[15] Y. Chen, L. Tai, K. Sun, and M. Li, "Monopair: Monocular 3d object detection using pairwise spatial relationships," in Proceedings of the IEEE Conference on Computer Vision and Pattern Recognition, pp. 12090-12099, 2020.

[16] X. Ma, Z. Wang, H. Li, P. Zhang, W. Ouyang, and X. Fan, "Accurate monocular 3d object detection via color-embedded 3d reconstruction for autonomous driving," in Proceedings of the IEEE International Conference on Computer Vision, pp. 6850 6860, 2019.

[17] J. Ku, A. D. Pon, and S. L. Waslander, "Monocular 3d object detection leveraging accurate proposals and shape reconstruction," in Proceedings of the IEEE Conference on Computer Vision and Pattern Recognition, pp. 867-876, 2019.

[18] P. Li, X. Chen, and S. Shen, "Stereo R-CNN based 3d object detection for autonomous driving," in Proceedings of the IEEE Conference on Computer Vision and Pattern Recognition, pp. 7644-7652, 2019.

[19] Y. Chen, S. Liu, X. Shen, and J. Jia, "DSGN: Deep stereo geometry network for $3 \mathrm{~d}$ object detection," in Proceedings of the 
IEEE Conference on Computer Vision and Pattern Recognition, pp. 12536-12545, 2020.

[20] Y. Wang, W.-L. Chao, D. Garg, B. Hariharan, M. Campbell, and K. Q. Weinberger, "Pseudo-lidar from visual depth estimation: Bridging the gap in $3 \mathrm{~d}$ object detection for autonomous driving," in Proceedings of the IEEE Conference on Computer Vision and Pattern Recognition, pp. 8445-8453, 2019.

[21] X. Chen, K. Kundu, Z. Zhang, H. Ma, S. Fidler, and R. Urtasun, "Monocular 3d object detection for autonomous driving," in Proceedings of the IEEE Conference on Computer Vision and Pattern Recognition, pp. 2147-2156, 2016.

[22] B. Yang, W. Luo, and R. Urtasun, "Pixor: Real-time 3d object detection from point clouds," in Proceedings of the IEEE Conference on Computer Vision and Pattern Recognition, pp. 7652-7660, 2018.

[23] S. Shi, X. Wang, and H. Li, "Pointrenn: 3d object proposal generation and detection from point cloud," in Proceedings of the IEEE Conference on Computer Vision and Pattern Recognition, pp. 770-779, 2019.

[24] Z. Yang, Y. Sun, S. Liu, X. Shen, and J. Jia, "Std: Sparse-todense $3 \mathrm{~d}$ object detector for point cloud," in Proceedings of the IEEE International Conference on Computer Vision, pp. 19511960, 2019.

[25] Y. Chen, S. Liu, X. Shen, and J. Jia, "Fast point R-CNN," in Proceedings of the IEEE International Conference on Computer Vision, pp. 9774-9783, 2019.

[26] C. R. Qi, H. Su, K. Mo, and L. J. Guibas, "Pointnet: Deep learning on point sets for 3d classification and segmentation," in Proceedings of the IEEE Conference on Computer Vision and Pattern Recognition, pp. 77-85, 2017.

[27] S. Shi, C. Guo, L. Jiang, Z. Wang, J. Shi, X. Wang, and H. Li, "PV-RCNN: Point-voxel feature set abstraction for 3d object detection," in Proceedings of the IEEE Conference on Computer Vision and Pattern Recognition, pp. 10526-10535, 2020.

[28] X. Chen, H. Ma, J. Wan, B. Li, and T. Xia, "Multi-view 3d object detection network for autonomous driving," in Proceedings of the IEEE Conference on Computer Vision and Pattern Recognition, pp. 6526-6534, 2017.

[29] J. H. Yoo, Y. Kim, J. S. Kim, and J. W. Choi, "3d-cvf: Generating joint camera and lidar features using cross-view spatial feature fusion for 3d object detection," in European Conference on Computer Vision, pp. 720-736, 2020.

[30] C. Choy, J. Gwak, and S. Savarese, "4d spatio-temporal convnets: Minkowski convolutional neural networks," in Proceedings of the IEEE Conference on Computer Vision and Pattern Recognition, pp. 3075-3084, 2019.

[31] R. Huang, W. Zhang, A. Kundu, C. Pantofaru, D. A. Ross, T. Funkhouser, and A. Fathi, "An 1stm approach to temporal 3d object detection in lidar point clouds," in European Conference on Computer Vision, pp. 266-282, 2020.

[32] F. Scarselli, M. Gori, A. C. Tsoi, M. Hagenbuchner, and G. Monfardini, "The graph neural network model," IEEE Transactions on neural networks, vol. 20, no. 1, pp. 61-80, 2008.

[33] J. Bruna, W. Zaremba, A. Szlam, and Y. LeCun, "Spectral networks and locally connected networks on graphs," International Conference on Learning Representations, 2014.

[34] M. Henaff, J. Bruna, and Y. LeCun, "Deep convolutional networks on graph-structured data," arXiv preprint arXiv:1506.05163, 2015.

[35] M. Defferrard, X. Bresson, and P. Vandergheynst, "Convolutional neural networks on graphs with fast localized spectral filtering," in Advances in Neural Information Processing Systems, pp. 3837-3845, 2016.

[36] D. K. Hammond, P. Vandergheynst, and R. Gribonval, "Wavelets on graphs via spectral graph theory," Applied and Computational Harmonic Analysis, vol. 30, no. 2, pp. 129-150, 2011.

[37] M. Niepert, M. Ahmed, and K. Kutzkov, "Learning convolutional neural networks for graphs," in International Conference on Machine Learning, pp. 2014-2023, 2016.
[38] W. Hamilton, Z. Ying, and J. Leskovec, "Inductive representation learning on large graphs," in Advances in Neural Information Processing Systems, pp. 1024-1034, 2017.

[39] M. Schlichtkrull, T. N. Kipf, P. Bloem, R. Van Den Berg, I. Titov, and M. Welling, "Modeling relational data with graph convolutional networks," in European Semantic Web Conference. Springer, pp. 593-607, 2018.

[40] H. Gao and S. Ji, "Graph u-nets," in International Conference on Machine Learning, pp. 2083-2092, 2019.

[41] Y. Li, D. Tarlow, M. Brockschmidt, and R. Zemel, "Gated graph sequence neural networks," in International Conference on Learning Representations, 2016.

[42] S. Kearnes, K. McCloskey, M. Berndl, V. Pande, and P. Riley, "Molecular graph convolutions: moving beyond fingerprints," Journal of computer-aided molecular design, vol. 30, no. 8, pp. 595-608, 2016.

[43] V. Zayats and M. Ostendorf, "Conversation modeling on reddit using a graph-structured 1stm," Transactions of the Association for Computational Linguistics, vol. 6, pp. 121-132, 2018.

[44] N. Peng, H. Poon, C. Quirk, K. Toutanova, and W.-t. Yih, "Cross-sentence n-ary relation extraction with graph lstms," Transactions of the Association for Computational Linguistics, vol. 5, pp. 101-115, 2017.

[45] J. Gilmer, S. S. Schoenholz, P. F. Riley, O. Vinyals, and G. E. Dahl, "Neural message passing for quantum chemistry," in International Conference on Machine Learning, 2017, pp. 12631272.

[46] W. Wang, X. Lu, J. Shen, D. J. Crandall, and L. Shao, "Zero-shot video object segmentation via attentive graph neural networks," in Proceedings of the IEEE International Conference on Computer Vision, pp. 9235-9244, 2019.

[47] S. Qi, W. Wang, B. Jia, J. Shen, and S.-C. Zhu, "Learning human-object interactions by graph parsing neural networks," in Proceedings of the European Conference on Computer Vision, pp. 407-423, 2018.

[48] C. Si, Y. Jing, W. Wang, L. Wang, and T. Tan, "Skeletonbased action recognition with spatial reasoning and temporal stack learning," in Proceedings of the European Conference on Computer Vision, pp. 106-121, 2018.

[49] Q. Chen, L. Sun, Z. Wang, K. Jia, and A. Yuille, "Object as hotspots: An anchor-free 3d object detection approach via firing of hotspots," in European Conference on Computer Vision, pp. 68-84, 2020.

[50] D. Duvenaud, D. Maclaurin, J. Aguilera-Iparraguirre, R. Gómez-Bombarelli, T. Hirzel, A. Aspuru-Guzik, and R. P. Adams, "Convolutional networks on graphs for learning molecular fingerprints," in Advances in Neural Information Processing Systems, pp. 2224-2232, 2015.

[51] P. Battaglia, R. Pascanu, M. Lai, D. J. Rezende et al., "Interaction networks for learning about objects, relations and physics," in Advances in Neural Information Processing Systems, pp. 4502-4510, 2016.

[52] K. Cho, B. Van Merriënboer, C. Gulcehre, D. Bahdanau, F. Bougares, H. Schwenk, and Y. Bengio, in "Learning phrase representations using rnn encoder-decoder for statistical machine translation," Conference on Empirical Methods in Natural Language Processing, 2014.

[53] D. Bahdanau, K. Cho, and Y. Bengio, "Neural machine translation by jointly learning to align and translate," arXiv preprint arXiv:1409.0473, 2014.

[54] N. Srivastava, E. Mansimov, and R. Salakhudinov, "Unsupervised learning of video representations using lstms," in International Conference on Machine Learning, pp. 843-852, 2015.

[55] J. Chung, C. Gulcehre, K. Cho, and Y. Bengio, "Empirical evaluation of gated recurrent neural networks on sequence modeling," arXiv preprint arXiv:1412.3555, 2014.

[56] M. Liu and M. Zhu, "Mobile video object detection with temporally-aware feature maps," in Proceedings of the IEEE Conference on Computer Vision and Pattern Recognition, pp. 
5686-5695, 2018.

[57] C. Feichtenhofer, A. Pinz, and R. P. Wildes, "Spatiotemporal multiplier networks for video action recognition," in Proceedings of the IEEE Conference on Computer Vision and Pattern Recognition, pp. 7445-7454, 2017.

[58] Z. Gan, C. Gan, X. He, Y. Pu, K. Tran, J. Gao, L. Carin, and L. Deng, "Semantic compositional networks for visual captioning," in Proceedings of the IEEE Conference on Computer Vision and Pattern Recognition, pp. 1141-1150, 2017.

[59] N. Carion, F. Massa, G. Synnaeve, N. Usunier, A. Kirillov, and S. Zagoruyko, "End-to-end object detection with transformers," in European Conference on Computer Vision, pp. 213-229, 2020.

[60] K. He, X. Zhang, S. Ren, and J. Sun, "Deep residual learning for image recognition," in Proceedings of the IEEE Conference on Computer Vision and Pattern Recognition, pp. 770-778, 2016.

[61] J. Wang, S. Lan, M. Gao, and LS. Davis, "Infofocus: 3d object detection for autonomous driving with dynamic information modeling," in European Conference on Computer Vision, pp. 405-420, 2020.

[62] Y. Ye, H. Chen, C. Zhang, X. Hao, and Z. Zhang, "Sarpnet: Shape attention regional proposal network for lidar-based $3 \mathrm{~d}$ object detection," Neurocomputing, vol. 379, pp. 53-63, 2020.

[63] Z. Yang, Y. Sun, S. Liu, and J. Jia, "3DSSD: Point-Based 3D Single Stage Object Detector," in Proceedings of the IEEE Conference on Computer Vision and Pattern Recognition, pp. 11037-11045, 2020.

[64] S. Vora, A. H. Lang, B. Helou, and O. Beijbom, "Pointpainting: Sequential fusion for 3d object detection," in Proceedings of the IEEE Conference on Computer Vision and Pattern Recognition, pp. 4603-4611, 2020

[65] T. Wang, X. Zhu, and D. Lin, "Reconfigurable voxels: A new representation for lidar-based point clouds," arXiv preprint arXiv:2004.02724, 2020.

[66] P. Bhattacharyya, C. Huang, and K. Czarnecki, "Self-attention based context-aware $3 \mathrm{~d}$ object detection," arXiv preprint arXiv:2101.02672, 2021.

[67] X. Zhu, Y. Ma, T. Wang, Y. Xu, J. Shi, and D. Lin, "SSN: Shape signature networks for multi-class object detection from point clouds," arXiv preprint arXiv:2004.02774, 2020.

[68] B. Zhu, Z. Jiang, X. Zhou, Z. Li, and G. Yu, "Class-balanced grouping and sampling for point cloud $3 \mathrm{~d}$ object detection," arXiv preprint arXiv:1908.09492, 2019.

[69] Q. Chen, L. Sun, E. Cheung, and A. L. Yuille, "Every view counts: Cross-view consistency in $3 \mathrm{~d}$ object detection with hybrid-cylindrical-spherical voxelization," in Advances in Neural Information Processing Systems, vol. 33, 2020.

[70] M. Rapoport-Lavie and D. Raviv, "It's all around you: Rangeguided cylindrical network for 3d object detection," arXiv preprint arXiv:2012.03121, 2020.

[71] A. Geiger, P. Lenz, and R. Urtasun, "Are we ready for autonomous driving? the kitti vision benchmark suite," in Proceedings of the IEEE Conference on Computer Vision and Pattern Recognition, pp. 3354-3361, 2012.

[72] D. P. Kingma and J. Ba, "Adam: A method for stochastic optimization," arXiv preprint arXiv:1412.6980, 2014.

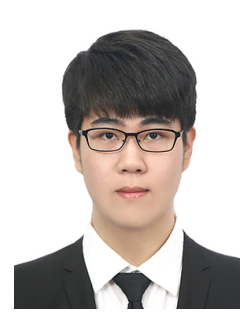

Junbo Yin is currently working toward the Ph.D. degree in the School of Computer Science, Beijing Institute of Technology, Beijing, China. His current research interests include Lidar-based 3D object detection and segmentation, selfsupervised learning, and visual object tracking.

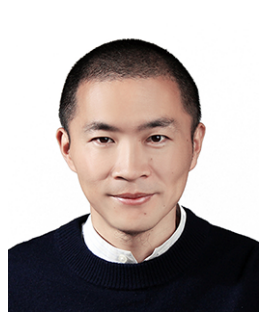

Jianbing Shen (M'11-SM'12) is currently acting as the Lead Scientist at the Inception Institute of Artificial Intelligence, Abu Dhabi, UAE. He is also an adjunct Professor with the School of Computer Science, Beijing Institute of Technology, Beijing, China. He has published more than 200 top journal and conference papers with Google Scholar Citations 10,800 times, and 18 papers are selected as the ESI Hightly Cited or ESI Hot Papers. His current research interests are in the areas of deep learning, computer vision, autonomous driving, video analysis, and intelligent systems. He is/was an Associate Editor of IEEE Trans. on Image Processing, IEEE Trans. on Neural Networks and Learning Systems, Pattern Recognition, and other journals.

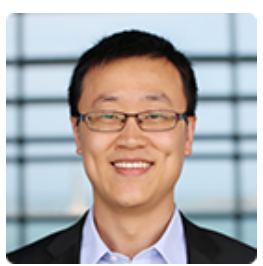

Xin Gao is currently a Full Professor of computer science with the Computer, Electrical and Mathematical Sciences and Engineering Division, King Abdullah University of Science and Technology (KAUST), Thuwal, Saudi Arabia. He is also the Associate Director of the Computational Bioscience Research Center, KAUST, and an Adjunct Faculty Member with the David R. Cheriton School of Computer Science, University of Waterloo. He received the Ph.D. degree in computer science from University of Waterloo, Waterloo, ON, Canada, in 2009. His group focuses on building computational models, developing machine learning methods, and designing efficient and effective algorithms, with particular a focus on applications to key open problems in biology. He has coauthored more than 200 research articles in the fields of machine learning and bioinformatics.

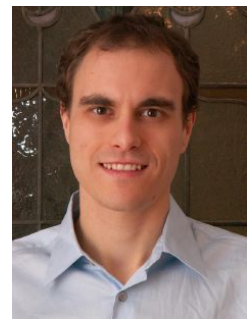

David Crandall is an Associate Professor in the School of Informatics and Computing, Indiana University. He received the Ph.D. degree in computer science from Cornell University in 2008, and the B.S. and M.S. degrees in computer science and engineering from Pennsylvania State University, State College in 2001. His research interests include computer vision, machine learning, and data mining. He is the recipient of a National Science Foundation CAREER Award and a Google Faculty Research Award. Currently, he is an Associate Editor of IEEE Transactions on Pattern Analysis and Machine Intelligence and IEEE Transactions on Multimedia.

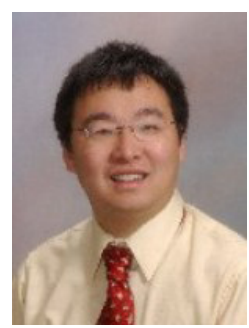

Ruigang Yang received the MS degree from Columbia University in 1998 and the $\mathrm{PhD}$ degree from the University of North Carolina, Chapel Hill in 2003. He is currently a Full professor of Computer Science at the University of Kentucky. His research interests span over computer vision and computer graphics, in particular in 3D reconstruction and $3 \mathrm{D}$ data analysis. He has published more than 100 papers, which, according to Google Scholar, has received close to 16,800 citations with an $\mathrm{h}$-index of 61 . He has received a number of awards, including the US National Science Foundation Faculty Early Career Development (CAREER) Program Award in 2004, best Demonstration Award at CVPR 2007 and the Deans Research Award at the University of Kentucky in 2013. He has served as Area Chairs for premium vision conferences (such as ICCV/CVPR), and served as a Program Chair for CVPR 2021. He is currently an associate editor of the IEEE Transactions on Pattern Analysis and Machine Intelligence and a senior member of IEEE. 


\section{A brief summary of differences}

This paper builds upon our recent IEEE CVPR'20 conference paper [1], and the document summarizes the changes in our extended manuscript.

- In this work, we first provide a more general framework that works with both anchor-based and anchor-free detection settings. In this regard, our approach can be easily incorporated with leading 3D object detectors. On the contrary, our previous work [1] only supports 3D object detection in the online mode with an anchor-based detector.

- Second, we extend our method to both online and offline detection modes, which can benefit more applications with improved accuracy, such as acting as annotation tools.

- Third, this paper provides a more in-depth discussion on the algorithm with more details including its motivation, technical preliminary, network architecture and implementation.

- Fourth, extensive ablation studies are conducted to thoroughly and rigorously assess the broad effectiveness of our model.

- Last but not least, we empirically observe that the proposed model outperforms all the algorithms on the nuScenes leaderboard without any bells and whistles. 


\title{
LiDAR-based Online 3D Video Object Detection with Graph-based Message Passing and Spatiotemporal Transformer Attention
}

\author{
Junbo Yin ${ }^{1,2}$, Jianbing Shen ${ }^{1,4 *}$ Chenye Guan ${ }^{2,3}$, Dingfu Zhou ${ }^{2,3}$, Ruigang Yang ${ }^{2,3,5}$ \\ ${ }^{1}$ Beijing Lab of Intelligent Information Technology, School of Computer Science, Beijing Institute of Technology, China \\ ${ }^{2}$ Baidu Research $\quad{ }^{3}$ National Engineering Laboratory of Deep Learning Technology and Application, China \\ ${ }^{4}$ Inception Institute of Artificial Intelligence, UAE ${ }^{5}$ University of Kentucky, Kentucky, USA \\ \{yinjunbocn, shenjianbingcg\}@gmail.com https://github.com/yinjunbo/3DVID
}

\begin{abstract}
Existing LiDAR-based 3D object detectors usually focus on the single-frame detection, while ignoring the spatiotemporal information in consecutive point cloud frames. In this paper, we propose an end-to-end online $3 D$ video object detector that operates on point cloud sequences. The proposed model comprises a spatial feature encoding component and a spatiotemporal feature aggregation component. In the former component, a novel Pillar Message Passing Network (PMPNet) is proposed to encode each discrete point cloud frame. It adaptively collects information for a pillar node from its neighbors by iterative message passing, which effectively enlarges the receptive field of the pillar feature. In the latter component, we propose an Attentive Spatiotemporal Transformer GRU (AST-GRU) to aggregate the spatiotemporal information, which enhances the conventional ConvGRU with an attentive memory gating mechanism. AST-GRU contains a Spatial Transformer Attention (STA) module and a Temporal Transformer Attention (TTA) module, which can emphasize the foreground objects and align the dynamic objects, respectively. Experimental results demonstrate that the proposed $3 D$ video object detector achieves state-of-the-art performance on the large-scale nuScenes benchmark.
\end{abstract}

\section{Introduction}

LiDAR-based 3D object detection plays a critical role in a wide range of applications, such as autonomous driving, robot navigation and virtual/augmented reality [11, 46]. The majority of current 3D object detection approaches [42, $58,6,62,24]$ follow the single-frame detection paradigm, while few of them perform detection in the point cloud video. A point cloud video is defined as a temporal sequence of point cloud frames. For instance, in the nuScenes

\footnotetext{
${ }^{*}$ Corresponding author: Jianbing Shen.
}

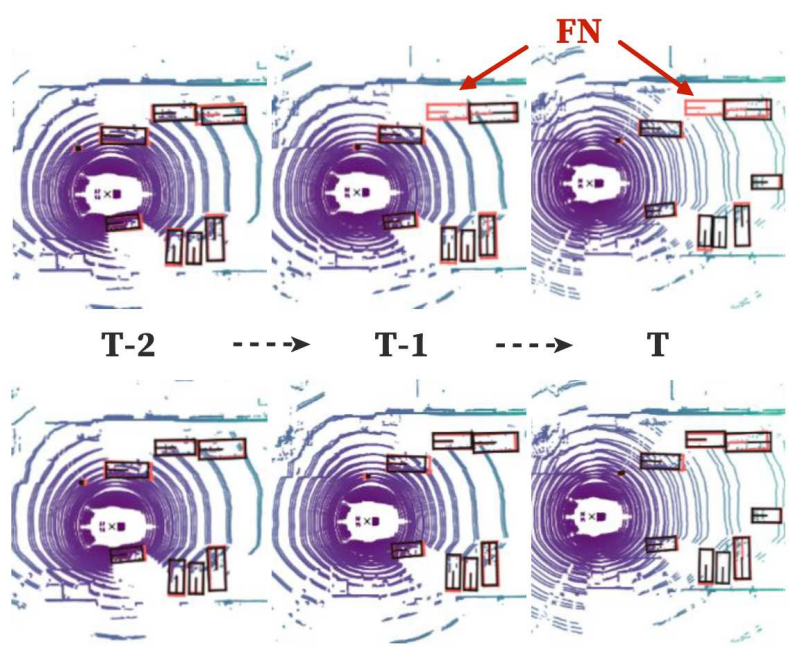

Figure 1: Occlusion situation in autonomous driving scenarios. Typical single-frame 3D object detector, e.g. [24], often leads to false-negative (FN) results (top row). In contrast, our online 3D video object detector can handle this (bottom row). The grey and red boxes denote the predictions and ground-truths, respectively.

dataset [4], 20 point cloud frames can be captured per second with a modern 32-beam LiDAR sensor. Detection in single frame may suffer from several limitations due to the sparse nature of point cloud. In particular, occlusions, longdistance and non-uniform sampling inevitably occur on a certain frame, where a single-frame object detector is incapable of handling these situations, leading to a deteriorated performance, as shown in Fig 1. However, a point cloud video contains rich spatiotemporal information of the foreground objects, which can be explored to improve the detection performance. The major concern of constructing a 3D video object detector is how to model the spatial and temporal feature representation for the consecutive point cloud frames. In this work, we propose to integrate a graph-based spatial feature encoding component with an attention-aware spatiotemporal feature aggregation component, to capture the video coherence in consecutive point 
cloud frames, which yields an end-to-end online solution for the LiDAR-based 3D video object detection.

Popular single-frame 3D object detectors tend to first discretize the point cloud into voxel or pillar girds [62, 56, 24], and then extract the point cloud features using stacks of convolutional neural networks (CNNs). Such approaches incorporate the success of existing 2D or 3D CNNs and usually gain better computational efficiency compared with the point-based methods [42, 37]. Therefore, in our spatial feature encoding component, we also follow this paradigm to extract features for each input frame. However, a potential problem with these approaches lies in that they only focus on a locally aggregated feature, i.e., employing a PointNet [39] to extract features for separate voxels or pillars as in [62] and [24]. To further enlarge the receptive fields, they have to apply the stride or pooling operations repeatedly, which will cause the loss of the spatial information. To alleviate this issue, we propose a novel graph-based network, named Pillar Message Passing Network (PMPNet), which treats a non-empty pillar as a graph node and adaptively enlarges the receptive field for a node by aggregating messages from its neighbors. PMPNet can mine the rich geometric relations among different pillar grids in a discretized point cloud frame by iteratively reasoning on a $k$-NN graph. This effectively encourages information exchanges among different spatial regions within a frame.

After obtaining the spatial features of each input frame, we assemble these features in our spatiotemporal feature aggregation component. Since ConvGRU [1] has shown promising performance in the 2D video understanding field, we suggest an Attentive Spatiotemporal Transformer GRU (AST-GRU) to extend ConvGRU to the 3D field through capturing dependencies of consecutive point cloud frames with an attentive memory gating mechanism. Specifically, there exist two potential limitations when considering the LiDAR-based 3D video object detection in autonomous driving scenarios. First, in the bird's eye view, most foreground objects (e.g., cars and pedestrians) occupy small regions, and the background noise is inevitably accumulated as computing the new memory in a recurrent unit. Thus, we propose to exploit the Spatial Transformer Attention (STA) module, an intra-attention derived from [48, 53], to suppress the background noise and emphasize the foreground objects by attending each pixel with the context information. Second, when updating the memory in the recurrent unit, the spatial features of the two inputs (i.e., the old memory and the new input) are not well aligned. In particular, though we can accurately align the static objects across frames using the ego-pose information, the dynamic objects with large motion are not aligned, which will impair the quality of the new memory. To address this, we propose a Temporal Transformer Attention (TTA) module that adaptively captures the object motions in consecutive frames with a temporal inter-attention mechanism. This will better utilize the modified deformable convolutional layers [65, 64]. Our AST-GRU can better handle the spatiotemporal features and produce a more reliable new memory, compared with the vanilla ConvGRU. To summarize, we propose a new LiDAR-based online 3D video object detector that leverages the previous long-term information to improve the detection performance. In our model, a novel PMPNet is introduced to adaptively enlarge the receptive field of the pillar nodes in a discretized point clod frame by iterative graph-based message passing. The output sequential features are then aggregated in the proposed AST-GRU to mine the rich coherence in the point cloud video by using an attentive memory gating mechanism. Extensive evaluations demonstrate that our 3D video object detector achieves better performance against the single-frame detectors on the large-scale nuScenes benchmark.

\section{Related Work}

LiDAR-based 3D Object Detection. Existing works on 3D object detection can be roughly categorized into three groups, which are LiDAR-based [42, 58, 62, 24, 61, 56], image-based [22, 54, 26, 34, 25] and multi-sensor fusionbased [5, 29, 30, 21, 38] methods. Here, we focus on the LiDAR-based approaches since they are less sensitive to different illumination and weather conditions. Among them, one category $[62,57,24]$ typically discretizes the point cloud into regular girds (e.g., voxels or pillars), and then exploits the 2D or 3D CNNs for features extraction. Another category [42, 58, 6] learns 3D representations directly from the original point cloud with a point-wise feature extractor like PointNet++ [39]. It is usually impractical to directly apply the point-based detectors in scenes with large-scale point clouds, for they tend to perform feature extraction for every single point. For instance, a keyframe in nuScenes dataset [4] contains 300,000 point clouds, which are densified by 10 non-keyframe LiDAR sweeps within $0.5 \mathrm{~s}$. Operating on point clouds with such a scale will lead to non-trivial computation cost and memory demand. In contrast, the voxel-based methods can tackle this kind of difficulty for they are less sensitive to the number of points. Zhou et al. [62] first apply the end-to-end CNNs for voxelbased 3D object detection. They propose to describe each voxel with a Voxel Feature Encoding (VFE) layer, and utilize cascade 3D and 2D CNNs to extract the deep features. Then a Region Proposal Network (RPN) is employed to obtain the final detection results. After that, Lang et al. [24] further extend [62] by projecting the point clouds to the the bird's eye view and encoding each discretized gird (named pillars) with a Pillar Feature Network (PFN).

Both the VFE layers and the PFN only take into account separate voxels or pillars when generating the grid-level representation, which ignores the information exchange in 


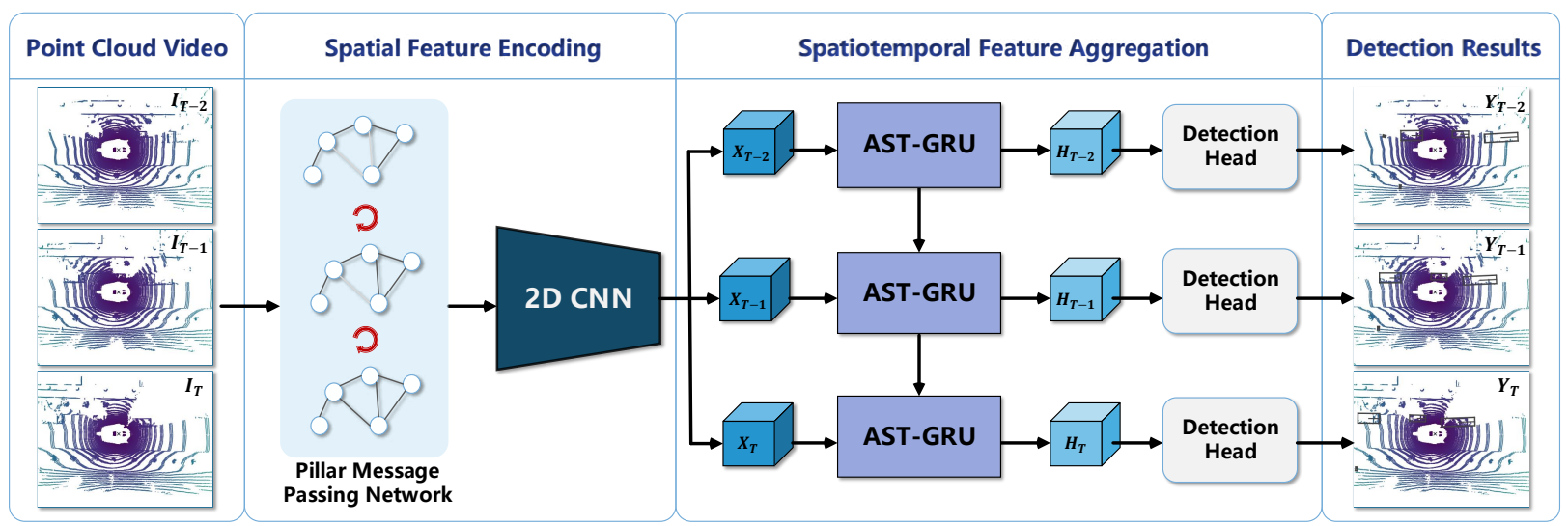

Figure 2: Our online 3D video object detection framework includes a spatial feature encoding component and a spatiotemporal feature aggregation component. In the former component, a novel PMPNet ( 33.1$)$ is proposed to extract the spatial features of each point cloud frame. Then, features from consecutive frames are sent to the AST-GRU ( $\$ 3.2)$ in the latter component, to aggregate the spatiotemporal information with an attentive memory gating mechanism.

larger spatial regions. In contrast, our PMPNet encodes the pillar feature from a global perspective by graph-based message passing, and thus promotes the representation with the non-local property. Besides, all these single-frame 3D object detectors can only process the point cloud data frameby-frame, lacking the exploration of the temporal information. Though [33] applies temporal 3D ConvNet on point cloud sequences, it encounters the feature collapse issue when downsampling the features in the temporal domain. Moreover, it cannot deal with long-term sequences with multi-frame labels. Our AST-GRU instead captures the long-term temporal information with an attentive memory gating mechanism, which can fully mine the spatiotemporal coherence in the point cloud video.

Graph Neural Networks. Graph Neural Networks (GNNs) are first introduced by Gori et al. [13] to model the intrinsic relationships of the graph-structured data. Then Scarselli et al. [41] extend it to different types of graphs. Afterward, GNNs are explored in two directions in terms of different message propagation strategies. The first group [28, $19,60,36,40]$ uses the gating mechanism to enable the information to propagate across the graph. For instance, Li et al. [28] leverage the recurrent neural networks to describe the state of each graph node. Then, Gilmer et al. [12] generalizes a framework to formulate the graph reasoning as a parameterized message passing network. Another group [3, 15, 9, 17, 27] integrates convolutional networks to the graph domain, named as Graph Convolutional Neural Networks (GCNNs), which update node features via stacks of graph convolutional layers. GNNs have achieved promising results in many areas $[9,10,51,2,52]$ due to the great expressive power of graphs. Our PMPNet belongs to the first group by capturing the pillar features with a gated message passing strategy, which is used to construct the spatial representation for each point cloud frame.

\section{Model Architecture}

In this section, we elaborate on our online $3 \mathrm{D}$ video object detection framework. As shown in Fig. 2, it consists of a spatial feature encoding component and a spatiotemporal feature aggregation component. Given the input sequences $\left\{\boldsymbol{I}_{t}\right\}_{t=1}^{T}$ with $T$ frames, we first convert the point cloud coordinates from the previous frames $\left\{\boldsymbol{I}_{t}\right\}_{t=1}^{T-1}$ to the current frame $\boldsymbol{I}_{T}$ using the GPS data, so as to eliminate the influence of the ego-motion and align the static objects across frames. Then, in the spatial feature encoding component, we extract features for each frame with the Pillar Message Passing Network (PMPNet) (§3.1) and a 2D backbone, producing sequential features $\left\{\boldsymbol{X}_{t}\right\}_{t=1}^{T}$. After that, these features are fed into the Attentive Spatiotemporal Transformer Gated Recurrent Unit (AST-GRU) (§3.2) in the spatiotemporal feature aggregation component, to generate the new memory features $\left\{\boldsymbol{H}_{t}\right\}_{t=1}^{T}$. Finally, a RPN head is applied on $\left\{\boldsymbol{H}_{t}\right\}_{t=1}^{T}$ to give the final detection results $\left\{\boldsymbol{Y}_{t}\right\}_{t=1}^{T}$. Some network architecture details are provided in $\S 3.3$.

\subsection{Pillar Message Passing Network}

Previous point cloud encoding layers (e.g., the VFE layers in [62] and the PFN in [24]) for voxel-based 3D object detection typically encode each voxel or pillar separately, which limits the expressive power of the grid-level representation due to the small receptive field of each local grid region. Our PMPNet instead seeks to explore the rich spatial relations among different gird regions by treating the non-empty pillar grids as graph nodes. Such design effectively reserves the non-Euclidean geometric characteristics of the original point clouds and enhance the output pillar features with a non-locality property.

Given an input point cloud frame $\boldsymbol{I}_{t}$, we first uniformly discretize it into a set of pillars $\mathcal{P}$, with each pillar uniquely associated with a spatial coordinate in the $x-y$ plane as 

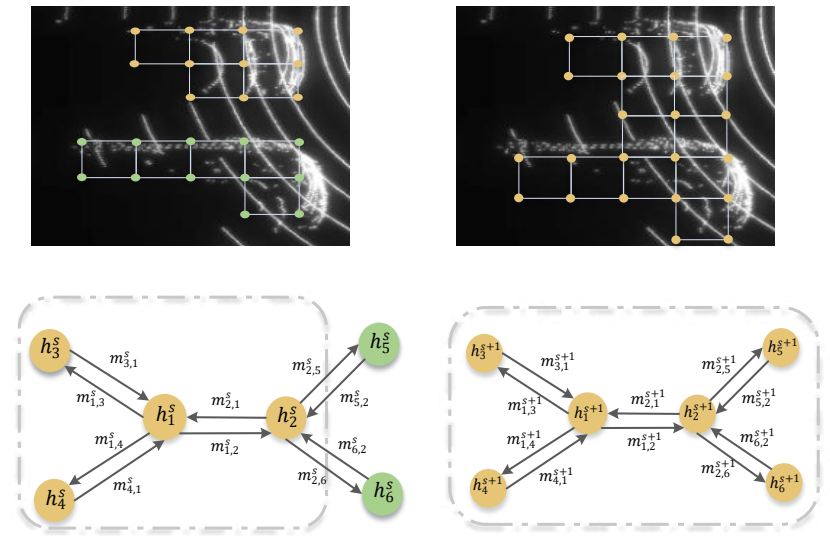

Figure 3: Illustration of one iteration step for message propagation, where $h_{i}$ is the state of node $v_{i}$. In step $s$, the neighbors for $h_{1}$ are $\{h 2, h 3, h 4\}$ (within the gray dash line), presenting the pillars in the top car. After aggregating messages from the neighbors, the receptive field of $h_{1}$ is enlarged in step $s+1$, indicating the relations with nodes from the bottom car are modeled.

in [24]. Then, PMPNet maps the resultant pillars to a directed graph $\mathcal{G}=(\mathcal{V}, \mathcal{E})$, where node $v_{i} \in \mathcal{V}$ represents a non-empty pillar $P_{i} \in \mathcal{P}$ and edge $e_{i, j} \in \mathcal{E}$ indicates the message passed from node $v_{i}$ to $v_{j}$. For reducing the computational overhead, we define $\mathcal{G}$ as a $k$-nearest neighbor $(k$-NN) graph, which is built from the geometric space by comparing the centroid distance among different pillars.

To explicitly mine the rich relations among different pillar nodes, PMPNet performs iterative message passing on $\mathcal{G}$ and updates the nodes state at each iteration step. Concretely, given a node $v_{i}$, we first utilize a pillar feature network (PFN) [24] to describe its initial state $\boldsymbol{h}_{i}^{0}$ at iteration step $s=0$ :

$$
\boldsymbol{h}_{i}^{0}=F_{\mathrm{PFN}}\left(P_{i}\right) \in \mathbb{R}^{L},
$$

where $\boldsymbol{h}_{i}^{0}$ is a $L$-dim vector and $P_{i} \in \mathbb{R}^{N \times D}$ presents a pillar containing $N$ LiDAR points, with each point parameterized by $D$ dimension representation (e.g., the XYZ coordinates and the received reflectance). The PFN is realized by applying fully connected layers on each point within the pillar, then summarizing features of all points through a channel-wise maximum operation. The initial node state $\boldsymbol{h}_{i}^{0}$ is a locally aggregated feature, only including points information within a certain pillar grid.

Next, we elaborate on the message passing process. One iteration step of message propagation is illustrated in Fig. 3. At step $s$, a node $v_{i}$ aggregates information from all the neighbor nodes $v_{j} \in \boldsymbol{\Omega}_{v_{i}}$ in the $k$-NN graph. We define the incoming edge feature from node $v_{j}$ as $\boldsymbol{e}_{j, i}^{s}$, indicating the relation between node $v_{i}$ and $v_{j}$. Inspired by [55], the incoming edge feature $e_{j, i}^{s}$ is given by:

$$
\boldsymbol{e}_{j, i}^{s}=\boldsymbol{h}_{j}^{s}-\boldsymbol{h}_{i}^{s} \in \mathbb{R}^{L},
$$

which is an asymmetric function encoding the local neigh- bor information. Accordingly, we have the message passed from $v_{j}$ to $v_{i}$, which is denoted as:

$$
\boldsymbol{m}_{j, i}^{s+1}=\phi_{\theta}\left(\left[\boldsymbol{h}_{i}^{s}, \boldsymbol{e}_{j, i}^{s}\right]\right) \in \mathbb{R}^{L^{\prime}},
$$

where $\phi_{\theta}$ is parameterized by a fully connected layer, which takes as input the concatenation of $\boldsymbol{h}_{i}^{s}$ and $\boldsymbol{e}_{j, i}^{s}$, and yields a $L^{\prime}$-dim feature.

After computing all the pair-wise relations between $v_{i}$ and the neighbors $v_{j} \in \boldsymbol{\Omega}_{v_{i}}$ of , we summarize the received $k$ messages with a maximum operation:

$$
\boldsymbol{m}_{i}^{s+1}=\max _{j \in \boldsymbol{\Omega}_{i}}\left(\boldsymbol{m}_{j, i}^{s+1}\right) \in \mathbb{R}^{L^{\prime}},
$$

Then, we update the node state $\boldsymbol{h}_{i}^{s}$ with $\boldsymbol{h}_{i}^{s+1}$ for node $v_{i}$. The update process should consider both the newly collected message $\boldsymbol{m}_{i}^{s+1}$ and the previous state $\boldsymbol{h}_{i}^{s}$. Recurrent neural network and its variants $[16,47]$ can adaptively capture dependencies in different time steps. Hence, we utilize Gated Recurrent Unit (GRU) [7] as the update function for its better convergence characteristic. The update process is then formulated as follows:

$$
\boldsymbol{h}_{i}^{s+1}=\operatorname{GRU}\left(\boldsymbol{h}_{i}^{s}, \boldsymbol{m}_{i}^{s+1}\right) \in \mathbb{R}^{L},
$$

In this way, the new node state $\boldsymbol{h}_{i}^{s+1}$ contains the information from all the neighbor nodes of $v_{i}$. Moreover, a neighbor node $v_{j}$ also collects information from its own neighbors $\boldsymbol{\Omega}_{v_{j}}$. Consequently, after the totally $S$ iteration steps, node $v_{i}$ is able to aggregate information from the high-order neighbors. This effectively enlarges the perceptual range for each pillar grid and enables our model to better recognize objects from a global view.

Note that each pillar corresponds with a spatial coordinate in the $x-y$ plane. Therefore, after performing the iterative message passing, the encoded pillar features are then scattered back as a 3D tensor $\tilde{\boldsymbol{I}}_{t} \in \mathbb{R}^{W \times H \times C}$, which can be further exploited by the $2 \mathrm{D}$ CNNs. Here, we leverage the backbone network in [62] to further extract features for $\tilde{\boldsymbol{I}}_{t}$ :

$$
\boldsymbol{X}_{t}=F_{\mathrm{B}}\left(\tilde{\boldsymbol{I}}_{t}\right) \in \mathbb{R}^{w \times h \times c},
$$

where $F_{\mathrm{B}}$ denotes the backbone network and $\boldsymbol{X}_{t}$ is the spatial features of $\boldsymbol{I}_{t}$. Details of the PMPNet and the backbone network can be found in $\S 3.3$.

\subsection{Attentive Spatiotemporal Transformer GRU}

Since the sequential features $\left\{\boldsymbol{X}_{t}\right\}_{t=1}^{T}$ produced by the spatial feature encoding component are regular tensors, we can employ the ConvGRU [1] to fuse these features in our spatiotemporal feature aggregation component. However, it may suffer from two limitations when directly applying the ConvGRU. On the one hand, the interest objects are relatively small in the bird's eye view compared with those 
in the 2D images (e.g., an average of $18 \times 8$ pixels for cars with the pillar size of $0.25^{2} \mathrm{~m}^{2}$ ). This may cause the background noise to dominate the results when computing the memory. On the other hand, though the static objects can be well aligned across frames using the GPS data, the dynamic objects with large motion still lead to an inaccurate new memory. To address the above issues, we propose the AST-GRU to equip the vanilla ConvGRU [1] with a spatial transformer attention (STA) module and a temporal transformer attention (TTA) module. As illustrated in Fig. 4, the STA module stresses the foreground objects in $\left\{\boldsymbol{X}_{t}\right\}_{t=1}^{T}$ and produces the attentive new input $\left\{\boldsymbol{X}_{t}^{\prime}\right\}_{t=1}^{T}$, while the TTA module aligns the dynamic objects in $\left\{\boldsymbol{H}_{t-1}\right\}_{t=1}^{T}$ and $\left\{\boldsymbol{X}_{t}^{\prime}\right\}_{t=1}^{T}$, and outputs the attentive old memory $\left\{\boldsymbol{H}_{t-1}^{\prime}\right\}_{t=1}^{T}$. Then, $\left\{\boldsymbol{X}_{t}^{\prime}\right\}_{t=1}^{T}$ and $\left\{\boldsymbol{H}_{t-1}^{\prime}\right\}_{t=1}^{T}$ are used to generate the new memory $\left\{\boldsymbol{H}_{t}\right\}_{t=1}^{T}$, and further produce the final detections $\left\{\boldsymbol{Y}_{t}\right\}_{t=1}^{T}$. Before giving the details of the STA and TTA modules, we first review the vanilla ConvGRU.

Vanilla ConvGRU. GRU model [7] operates on a sequence of inputs to adaptively capture the dependencies in different time steps with a memory mechanism. ConvGRU is a variant of the conventional GRU model, which employs convolution operations rather than the fully connected ones, to reduce the number of parameters and preserve the spatial resolution of the input features. ConvGRU has made promising results on many tasks [32, 49, 23, 50], and has shown better results than the LSTM [43] counterparts in terms of the convergence time [8]. More specifically, ConvGRU contains an update gate $\boldsymbol{z}_{t}$, a reset gate $\boldsymbol{r}_{t}$, a candidate memory $\tilde{\boldsymbol{H}}_{t}$ and a new memory $\boldsymbol{H}_{t}$. At each time step, the new memory $\boldsymbol{H}_{t}$ (also named as the hidden state) is computed based on the old memory $\boldsymbol{H}_{t-1}$ and the new input $\boldsymbol{X}_{t}$, which can be denoted by the following equations:

$$
\begin{aligned}
\boldsymbol{z}_{t} & =\sigma\left(\boldsymbol{W}_{z} * \boldsymbol{X}_{t}+\boldsymbol{U}_{z} * \boldsymbol{H}_{t-1}\right), \\
\boldsymbol{r}_{t} & =\sigma\left(\boldsymbol{W}_{r} * \boldsymbol{X}_{t}+\boldsymbol{U}_{r} * \boldsymbol{H}_{t-1}\right), \\
\tilde{\boldsymbol{H}}_{t} & =\tanh \left(\boldsymbol{W} * \boldsymbol{X}_{t}+\boldsymbol{U} *\left(\boldsymbol{r}_{t} \circ \boldsymbol{H}_{t-1}\right)\right), \\
\boldsymbol{H}_{t} & =\left(\mathbf{1}-\boldsymbol{z}_{t}\right) \circ \boldsymbol{H}_{t-1}+\boldsymbol{z}_{t} \circ \tilde{\boldsymbol{H}}_{t},
\end{aligned}
$$

where ' $*$ ' and ' $\circ$ ' denote the convolution operation and Hadamard product, and $\sigma$ is a sigmoid function. $\boldsymbol{W}, \boldsymbol{W}_{z}, \boldsymbol{W}_{r}$ and $\boldsymbol{U}, \boldsymbol{U}_{z}, \boldsymbol{U}_{r}$ are the $2 \mathrm{D}$ convolutional kernels. When computing the candidate memory $\tilde{\boldsymbol{H}}_{t}$, the importance of the old memory $\boldsymbol{H}_{t-1}$ and the new input $\boldsymbol{X}_{t}$ is determined by the reset gate $\boldsymbol{r}_{t}$, i.e., the information of $\tilde{\boldsymbol{H}}_{t}$ all comes from $\boldsymbol{X}_{t}$ when $\boldsymbol{r}_{t}=0$. Additionally, the update gate $z_{t}$ decides the degree to which the unit accumulates the old memory $\boldsymbol{H}_{t-1}$, to yield the new memory $\boldsymbol{H}_{t}$. In $\S 4.2$, we show that the vanilla ConvGRU has outperformed the simple point cloud merging [4] and the temporal 3D ConvNet [33]. Next, we present how we promote the vanilla ConvGRU with the STA and TTA modules.

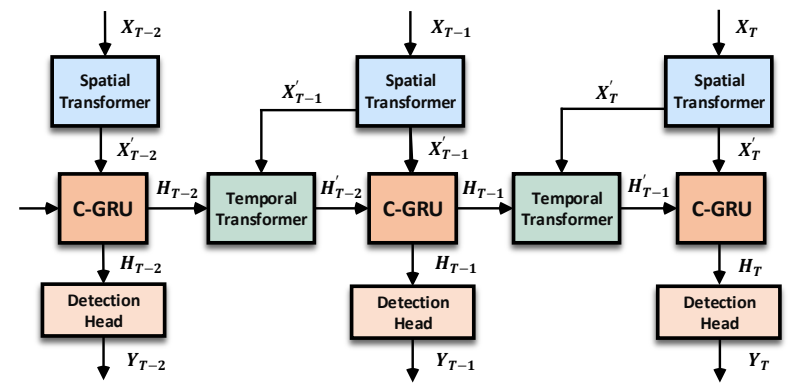

Figure 4: The detailed architecture of the proposed ASTGRU, which consists of a spatial transformer attention (STA) module and a temporal transformer attention (TTA) module. ASTGRU models the dependencies of consecutive frames and produces the attentive new memory $\left\{\boldsymbol{H}_{t}\right\}_{t=1}^{T}$.

Spatial Transformer Attention. The core idea of the STA module is to attend each pixel-level feature $\boldsymbol{x} \in \boldsymbol{X}_{t}$ with a rich spatial context, to better distinguish a foreground object from the background noise. Basically, a transformer attention receives a query $\boldsymbol{x}_{q} \in \boldsymbol{X}_{t}$ and a set of keys $\boldsymbol{x}_{k} \in \boldsymbol{\Omega}_{\boldsymbol{x}_{q}}$ (e.g., the neighbors of $\boldsymbol{x}_{q}$ ), to calculate an attentive output $\boldsymbol{y}_{q}$. The STA is designed as an intra-attention, which means both the query and key are from the same input feature $\boldsymbol{X}_{t}$.

Formally, given a query $\boldsymbol{x}_{q} \in \boldsymbol{X}_{t}$ at location $q \in w \times h$, the attentive output $\boldsymbol{y}_{q}$ is computed by:

$$
\boldsymbol{y}_{q}=\sum_{k \in \boldsymbol{\Omega}_{q}} A\left(\phi_{Q}\left(\boldsymbol{x}_{q}\right), \phi_{K}\left(\boldsymbol{x}_{k}\right)\right) \circ \phi_{V}\left(\boldsymbol{x}_{k}\right),
$$

where $A(\cdot, \cdot)$ is the attention weight. $\phi_{K}, \phi_{Q}$ and $\phi_{V}$ are the linear layers that map the inputs $\boldsymbol{x}_{q}, \boldsymbol{x}_{k} \in \boldsymbol{X}_{t}$ into different embedding subspaces. The attention weight $A(\cdot, \cdot)$ is computed from the embedded query-key pair $\left(\phi_{Q}\left(\boldsymbol{x}_{q}\right), \phi_{K}\left(\boldsymbol{x}_{k}\right)\right)$, and is then applied to the neighbor values $\phi_{V}\left(\boldsymbol{x}_{k}\right)$.

Since we need to obtain the attention for all the querykey pairs, the linear layers, $\phi_{K}, \phi_{Q}$ and $\phi_{V}$, are then achieved by the convolutional layers, $\Phi_{K}, \Phi_{Q}$ and $\Phi_{V}$, to facilitate the computation. Specifically, the input features $\boldsymbol{X}_{t}$ are first embedded as $\boldsymbol{K}_{t}, \boldsymbol{Q}_{t}$ and $\boldsymbol{V}_{t} \in \mathbb{R}^{w \times h \times c^{\prime}}$ through $\Phi_{K}, \Phi_{Q}$ and $\Phi_{V}$. Then, we adjust the tensor shapes of $\boldsymbol{K}_{t}$ and $\boldsymbol{Q}_{t}$ to $l \times c^{\prime}$, where $l=w \times h$, in order to compute the attention weight:

$$
\tilde{\boldsymbol{A}}=\operatorname{softmax}\left(\boldsymbol{Q}_{t} \cdot \boldsymbol{K}_{t}^{T}\right) \in \mathbb{R}^{l \times l}
$$

where $A(\cdot, \cdot)$ is realized as a softmax layer to normalize the attention weight matrix. After that, $\tilde{\boldsymbol{A}}$ is employed to aggregate information from the values $\boldsymbol{V}_{t}$ through a matrix multiplication, generating the attentive output $\tilde{\boldsymbol{A}} \cdot \boldsymbol{V}_{t}$, with the tensor shape recovered to $w \times h \times c^{\prime}$. Finally, we obtain the spatially enhanced features $\boldsymbol{X}_{t}^{\prime}$ through a residual operation [14], which can be summarized as:

$$
\boldsymbol{X}_{t}^{\prime}=\boldsymbol{W}_{\text {out }} *\left(\tilde{\boldsymbol{A}} \cdot \boldsymbol{V}_{t}\right)+\boldsymbol{X}_{t} \in \mathbb{R}^{w \times h \times c}
$$


where $\boldsymbol{W}_{\text {out }}$ is the output layer of the attention head that maps the embedding subspace $\left(c^{\prime}-\operatorname{dim}\right)$ of $\tilde{\boldsymbol{A}} \cdot \boldsymbol{V}_{t}$ back to the original space (c-dim). In this way, $\boldsymbol{X}_{t}^{\prime}$ contains the information from its spatial context and thus can better focus on the meaningful foreground objects.

Temporal Transformer Attention. To adaptively align the features of dynamic objects from $\boldsymbol{H}_{t-1}$ to $\boldsymbol{X}_{t}^{\prime}$, we apply the modified deformable convolutional layers $[65,64]$ as a special instantiation of the transformer attention. The core is to attend the queries in $\boldsymbol{H}_{t-1}$ with adaptive supporting key regions computed by integrating the motion information.

Specifically, given a vanilla deformable convolutional layer with kernel size $3 \times 3$, let $\boldsymbol{w}_{m}$ denotes the learnable weights, and $p_{m} \in\{(-1,-1),(-1,0), \ldots,(1,1)\}$ indicates the predetermined offset in total $M=9$ grids. The output $\boldsymbol{h}_{q}^{\prime}$ for input $\boldsymbol{h}_{q} \in \boldsymbol{H}_{t-1}$ at location $q \in w \times h$ can be expressed as:

$$
\boldsymbol{h}_{q}^{\prime}=\sum_{m=1}^{M} \boldsymbol{w}_{m} \cdot \boldsymbol{h}_{q+p_{m}+\Delta p_{m}}
$$

where $\Delta p_{m}$ is the deformation offset learnt through a separate regular convolutional layer $\Phi_{R}$, i.e., $\Delta p_{m} \in \Delta \boldsymbol{P}_{t-1}=$ $\Phi_{R}\left(\boldsymbol{H}_{t-1}\right) \in \mathbb{R}^{w \times h \times 2 r^{2}}$, where the channel number $2 r^{2}$ denotes the offsets in the X-y plane for the $r \times r$ convolutional kernel. We can also reformulate Eq. 14 from the perspective of transformer attention as in Eq. 11, such that the attentive output $\boldsymbol{h}_{q}^{\prime}$ of query $\boldsymbol{h}_{q}$ is given by:

$$
\boldsymbol{h}_{q}^{\prime}=\sum_{m=1}^{M} \boldsymbol{w}_{m} \cdot \sum_{k \in \boldsymbol{\Omega}_{q}} G\left(k, q+p_{m}+\Delta p_{m}\right) \cdot \phi_{\mathrm{V}}\left(\boldsymbol{h}_{k}\right),
$$

where $\phi_{\mathrm{V}}$ is an identity function, and $\boldsymbol{w}_{m}$ acts as the weights in different attention heads [64], with each head corresponding to a sampled key position $k \in \boldsymbol{\Omega}_{q} . G(\cdot, \cdot)$ is the attention weight defined by a bilinear interpolation function, such that $G(a, b)=\max (0,1-|a-b|)$.

The supporting key regions $\boldsymbol{\Omega}_{q}$ play an important role in attending $\boldsymbol{h}_{q}$, which are determined by the deformation offset $\Delta p_{m} \in \Delta \boldsymbol{P}_{t-1}$. In our TTA module, we compute $\Delta \boldsymbol{P}_{t-1}$ not only through $\boldsymbol{H}_{t-1}$, but also through a motion map, which is defined as the difference of $\boldsymbol{H}_{t-1}$ and $\boldsymbol{X}_{t}^{\prime}$ :

$$
\Delta \boldsymbol{P}_{t-1}=\Phi_{R}\left(\left[\boldsymbol{H}_{t-1}, \boldsymbol{H}_{t-1}-\boldsymbol{X}_{t}^{\prime}\right]\right) \in \mathbb{R}^{w \times h \times 2 r^{2}},
$$

where $\Phi_{R}$ is a regular convolutional layer with the same kernel size as that in the deformable convolutional layer, and $[\cdot, \cdot]$ is the concatenation operation. The intuition is that, in the motion map, the features response of the static objects is very low since they have been spatially aligned in $\boldsymbol{H}_{t-1}$ and $\boldsymbol{X}_{t}^{\prime}$, while the features response of the dynamic objects remains high. Therefore, we integrate $\boldsymbol{H}_{t-1}$ with the motion map, to further capture the motions of dynamic objects. Then, $\Delta \boldsymbol{P}_{t-1}$ is used to select the supporting key regions and further attend $\boldsymbol{H}_{t-1}$ for all the query regions $q \in w \times h$ in terms of Eq. 15, yielding a temporally attentive memory $\boldsymbol{H}_{t-1}^{\prime}$. Since the supporting key regions are computed from both $\boldsymbol{H}_{t-1}$ and $\boldsymbol{X}_{t}^{\prime}$, our TTA module can be deemed as an inter-attention.

Additionally, we can stack multiple modified deformable convolutional layers to get a more accurate $\boldsymbol{H}_{t-1}^{\prime}$. In our implementation, we adopt two layers. The latter layer takes as input $\left[\boldsymbol{H}_{t-1}^{\prime}, \boldsymbol{H}_{t-1}^{\prime}-\boldsymbol{X}_{t}^{\prime}\right]$ to predict the deformation offset according to Eq. 16, and the offset is then used to attend $\boldsymbol{H}_{t-1}^{\prime}$ via Eq. 15. Accordingly, we can now utilize the temporally attentive memory $\boldsymbol{H}_{t-1}^{\prime}$ and the spatially attentive input $\boldsymbol{X}_{t}^{\prime}$ to compute the new memory $\boldsymbol{H}_{t}$ in the recurrent unit (see Fig. 4). Finally, a RPN detection head is applied on $\boldsymbol{H}_{t}$ to produce the final detection results $\boldsymbol{Y}_{t}$.

\subsection{Network Details}

PMPNet. Our PMPNet is an end-to-end differentiable model achieved by parameterizing all the functions with neural networks. Given a discretized point cloud frame $\boldsymbol{I}_{t} \in \mathbb{R}^{P \times N \times D}$ with $P$ pillar nodes, $F_{\mathrm{PFN}}$ is first used to generates the initial node state $G^{0} \in \mathbb{R}^{P \times L}$ for all the nodes (Eq. 1), which is realized by a $1 \times 1$ convolutional layer followed by a max pooling layer that operates on the $N$ points. In each iteration step $s$, the edge features from the $K$ neighbor nodes are first collected as $\tilde{G}^{s} \in \mathbb{R}^{P \times K \times 2 L}$ (Eq. 2) with a concatenation operation. Then the message functions (Eq. 3 and Eq. 4) map the collected features $\tilde{G}^{s}$ to $M^{s} \in \mathbb{R}^{P \times L^{\prime}}$, through a $1 \times 1$ convolutional layer followed by a max pooling layer performing on the $K$ messages. The update function (Eq. 5) then updates the node state using a GRU with fully connected layers, by considering both the $G^{s} \in \mathbb{R}^{P \times L}$ and $M^{s} \in \mathbb{R}^{P \times L^{\prime}}$, and outputs $G^{s+1} \in \mathbb{R}^{P \times L}$. After $S$ iteration steps, we get the final node state $G^{S} \in \mathbb{R}^{P \times L}$, and scatter it back to a 3D tensor $\tilde{\boldsymbol{I}}_{t \in \mathbb{R}^{W \times H \times L}}$ (Eq. 6).

Backbone Module. As in [62], we utilize a 2D backbone network to further extract features for $\tilde{\boldsymbol{I}}_{t \in \mathbb{R}^{W \times H \times L} \text {, which }}$ consists of three blocks of fully convolutional layers. Each block is defined as a tuple $(S, Z, C)$. All the blocks have $Z \times Z$ convolutional kernels with output channel number $C$. The first layer of each block operates at stride $S$, while other layers have stride 1 . The output features of each block are resized to the same resolution via upsampling layers and then concatenated together to merge the semantic information from different feature levels.

AST-GRU Module. In our STA module, all the linear functions in Eq. 11 and $\boldsymbol{W}_{\text {out }}$ in Eq. 13 are $1 \times 1$ convolution layers. In our TTA module, the regular convolutional layers, the deformable convolutional layers and the ConvGRU all have learable kernels of size $3 \times 3$. 


\begin{tabular}{l||ccccccccccc}
\hline \multicolumn{1}{c|}{ Method } & Car & Pedestrian & Bus & Barrier & T.C. & Truck & Trailer & Moto. & Cons. & Bicycle & Mean \\
\hline VIPL_ICT [35] & 71.9 & 57.0 & 34.1 & 38.0 & 27.3 & 20.6 & 26.9 & 20.4 & 3.3 & 0.0 & 29.9 \\
MAIR [44] & 47.8 & 37.0 & 18.8 & 51.1 & 48.7 & 22.0 & 17.6 & 29.0 & 7.4 & $\mathbf{2 4 . 5}$ & 30.4 \\
PointPillars [24] & 68.4 & 59.7 & 28.2 & 38.9 & 30.8 & 23.0 & 23.4 & 27.4 & 4.1 & 1.1 & 30.5 \\
SARPNET [59] & 59.9 & 69.4 & 19.4 & 38.3 & 44.6 & 18.7 & 18.0 & 29.8 & 11.6 & 14.2 & 32.4 \\
WYSIWYG [18] & 79.1 & 65.0 & 46.6 & 34.7 & 28.8 & 30.4 & 40.1 & 18.2 & 7.1 & 0.1 & 35.0 \\
Tolist [35] & 79.4 & 71.2 & 42.0 & $\mathbf{5 1 . 2}$ & 47.8 & $\mathbf{3 4 . 5}$ & 34.8 & 36.8 & 9.8 & 12.3 & 42.0 \\
\hline Ours & $\mathbf{7 9 . 7}$ & $\mathbf{7 6 . 5}$ & $\mathbf{4 7 . 1}$ & 48.8 & $\mathbf{5 8 . 8}$ & 33.6 & $\mathbf{4 3 . 0}$ & $\mathbf{4 0 . 7}$ & $\mathbf{1 8 . 1}$ & 7.9 & $\mathbf{4 5 . 4}$ \\
\hline
\end{tabular}

Table 1: Quantitative detection results on the nuScenes 3D object detection benchmark. T.C. presents the traffic cone. Moto. and Cons. are short for the motorcycle and construction vehicle, respectively. Our 3D video object detector outperforms the single-frame detectors, achieving state-of-the-art performance on the leaderboard.

Detection Head. The detection head in [62] is applied on the attentive memory features. In particular, the smooth L1 loss and the focal loss [31] count for the object bounding box regression and classification, respectively. A corssentropy loss is used for the orientation classification. For the velocity regression required by the nuScenes benchmark, a simple L1 loss is adopted and shows substantial results.

\section{Experimental Results}

3D Video Object Detection Benchmark. We evaluate our algorithm on the challenging nuScenes 3D object detection benchmark [4], since the KITTI benchmark [11] does not provide the point cloud videos. nuScenes is a largescale dataset with a total of 1,000 scenes, where 700 scenes (28,130 samples) are for training and 150 scenes $(6,008$ samples) are for testing, resulting $7 \times$ as many annotations as the KITTI. The samples (also named as keyframes) in each video are annotated every $0.5 \mathrm{~s}$ with a full 360 -degree view, and their point clouds are densified by the 10 nonkeyframe sweeps frames, yielding around 300,000 point clouds with 5-dim representation $(x, y, z, r, \Delta t)$, where $r$ is the reflectance and $\Delta t$ describes the time lag to the keyframe (ranging from $0 \mathrm{~s}$ to $0.45 \mathrm{~s}$ ). Besides, nuScenes requires detecting objects for 10 classes with full 3D boxes, attributes and velocities.

Implementation Details. For each keyframe, we consider the point clouds within range of $[-50,50] \times[-50,50] \times$ $[-5,3]$ meters along the $\mathrm{X}, \mathrm{Y}$ and $\mathrm{Z}$ axes. The pillar resolution on the X-Y plane is $0.25^{2} \mathrm{~m}^{2}$. The pillar number $P$ used in PMPNet is 16,384, sampled from the total 25,000 pillars, with each pillar containing most $N=60$ points. The input point cloud is a $D=5$ dimensions representation $(x, y, z, r, \Delta t)$, which are then embedded into $L=L^{\prime}=64$ dimensions feature space after the total $S=3$ graph iteration steps. The convolutional kernels in the $2 \mathrm{D}$ backbone are of size $Z=3$ and the output channel number $C$ in each block is $(64,128,256)$. The upsampling layer has kernel size 3 and channel number 128. Thus, the final features map produced by the $2 \mathrm{D}$ backbone has a resolution of $100 \times 100 \times 384$. We calculate anchors for different classes using the mean sizes and set the matching threshold according to the class instance number. The coefficients of the loss functions for classification, localization and velocity prediction are set to 1,2 and 0.1 , respectively. NMS with IOU threshold 0.5 is utilized when generating the final detections. In both training and testing phases, we feed most 3 consecutive keyframes to the model due to the memory limitation. The training procedure has two stages. In the first stage, we pre-train the spatial features encoding component using the one-cycle policy [45] with a maximum learning rate of 0.003 . Then, we fix the learning rate to 0.0002 in the second stage to train the full model. We train 50 epochs for both stages with batch size 3. Adam optimizer [20] is used to optimize the loss functions.

\subsection{Quantitative and Qualitative Performance}

We present the performance comparison of our algorithm and other state-of-the-art approaches on the nuScenes benchmark in Table 1. PointPillars [24], SARPNET [59], WYSIWYG [18] and Tolist [35] are all voxel-based singleframe 3D object detectors. In particular, PointPillars is used as the baseline of our model. WYSIWYG is a recent algorithm that extends the PointPillars with a voxelized visibility map. Tolist uses a multi-head network that contains multiple prediction heads for different classes. Our 3D video object detector outperforms these approaches by a large margin. In particular, we improve the official PointPillars algorithm by $15 \%$. Please note that there is a severe class imbalance issue in the nuScenes dataset. The approach in [63] designs a class data augmentation algorithm. Further integrating with these techniques can promote the performance of our model. But we focus on exploring the spatiotemporal coherence in the point cloud video, and handling the class imbalance issue is not the purpose in this work. In addition, we further show some qualitative results in Fig. 5. Besides the occlusion situation in Fig. 1, we present another case of detecting the distant car (the car on the top right), whose point clouds are especially sparse, which is very challenging for the single-frame detectors. Again, our 3D video object detector effectively detects the distant car using the attentive temporal information. 

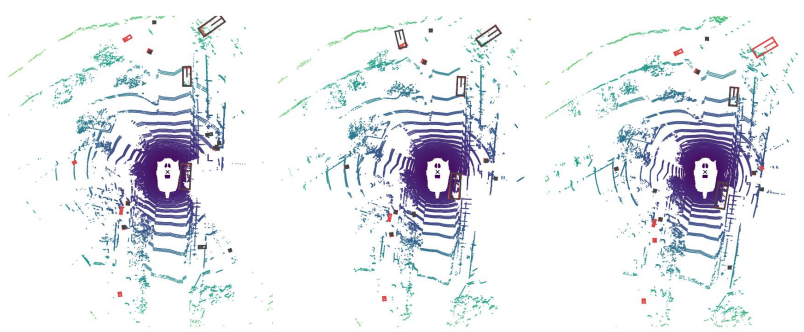

(a) Detection results from the single-frame 3D object detector [24].

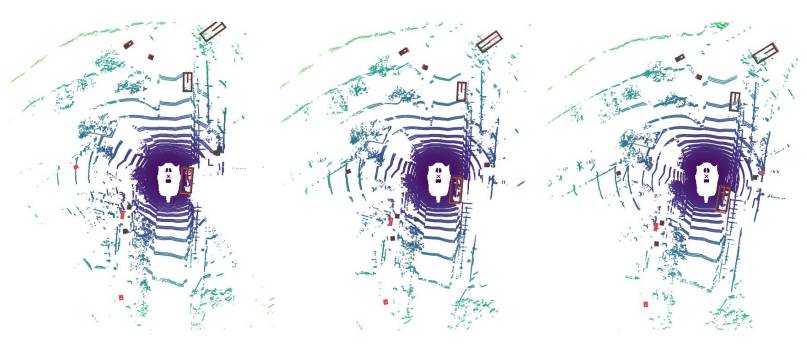

(b) Detection results from our 3D video object detector.

Figure 5: Detections for the distant cars. The grey and red boxes indicate the predictions and ground-truths, respectively.

\subsection{Ablation Study}

In this section, we investigate the effectiveness of each module in our algorithm. Since the training samples in the nuScenes is $7 \times$ as many as those in the KITTI $(28,130$ vs 3,712 ), it is non-trivial to train multiple models on the whole dataset. Hence, we use a mini train set for validation purposes. It contains around 3,500 samples uniformly sampled from the original train set. Besides, PointPillars [24] is used as the baseline detector in our model.

First, we evaluate our PMPNet in the spatial feature encoding component, by replacing the PFN in PointPillars with PMPNet. As shown in Table 2, it improves the baseline by $2.05 \%$. Second, we validate the ability of each module in the spatiotemporal feature aggregation component (i.e., ConvGRU, STA-GRU and TTA-GRU) through adding each module to the PointPillars. We can see that all these modules achieve better performance than the single-frame detector. Moreover, we compare AST-GRU with other video object detectors. Since each keyframe in nuScenes contains point clouds merged by previous 10 non-keyframe sweeps, the PointPillars baseline, trained on the merged keyframes, can be deemed as the simplest video object detector. Our AST-GRU improves it by $5.98 \%$. Then, we compare ASTGRU with the temporal 3D ConvNet-based method by implementing the late feature fusion module in [33]. Temporal 3D ConvNet can only access a single keyframe label $(0.5 \mathrm{~s})$ during training, and aggregating more labels instead impairs the performance. According to Table 2, the 3D ConvNetbased method surpasses the PointPillars baseline by $1.82 \%$.

\begin{tabular}{c|c|cc}
\hline \multirow{2}{*}{ Components } & \multirow{2}{*}{ Modules } & \multicolumn{2}{|c}{ Performance } \\
& & mAP & $\Delta$ \\
\hline Single-frame & PointPillars (PP) & 21.30 & - \\
3D Object Detector & PP + PMPNet & 23.35 & +2.05 \\
\hline \multirow{4}{*}{ 3D Video } & PP + 3D ConvNet & 23.12 & +1.82 \\
Object Detector & PP + ConvGRU & 23.83 & +2.53 \\
& PP + STA-GRU & 25.23 & +3.93 \\
& PP + TTA-GRU & 25.32 & +4.02 \\
& PP + AST-GRU & 27.28 & +5.98 \\
\cline { 2 - 4 } & Full Model & $\mathbf{2 9 . 3 5}$ & $\mathbf{+ 8 . 0 5}$ \\
\hline
\end{tabular}

Table 2: Ablation study for our 3D video object detector. PointPillars [24] is the reference baseline for computing the relative improvement $(\Delta)$.

\begin{tabular}{c|c|c|c}
\hline \multirow{2}{*}{ Aspect } & \multirow{2}{*}{ Modules } & \multicolumn{2}{|c}{ Performance } \\
& & mAP & \multicolumn{1}{|c}{$\Delta$} \\
\hline \multirow{4}{*}{$\begin{array}{c}\text { Input Lengths } \\
\text { (Full Model) }\end{array}$} & $\mathrm{T}=1$ & 16.84 & - \\
\cline { 2 - 4 } & $\mathrm{T}=2$ & 19.34 & +2.50 \\
\cline { 2 - 4 } & $\mathrm{T}=3$ & 20.27 & +3.43 \\
\cline { 2 - 4 } & $\mathrm{T}=4$ & 20.77 & +3.93 \\
\cline { 2 - 4 } & $\mathbf{T}=\mathbf{5}$ & $\mathbf{2 1 . 5 2}$ & $\mathbf{+ 4 . 6 8}$ \\
\hline
\end{tabular}

Table 3: Ablation study for the input lengths. Detection results with one input frame are used as the reference baseline.

In contrast, our AST-GRU further outperforms it by $4.16 \%$, which demonstrates the importance of the long-term temporal information. Finally, the full model with the PMPNet achieves the best performance.

Finally, we analyze the effect of the input sequence length. Since each keyframe contains quantities of point clouds that will increase the memory demand, we conduct this experiment without using the point clouds in nonkeyframe sweeps. Experimental results with different input lengths are shown in Table. 3, which demonstrates that using the previous long-term temporal information $(2.5 \mathrm{~s})$ can consistently gain better performance in 3D object detection.

\section{Conclusion}

This paper proposed a new 3D video object detector for exploring the spatiotemporal information in point cloud video. It has developed two new components: spatial feature encoding component and spatiotemporal feature aggregation component. We first introduce a novel PMPNet that considers the spatial features of each point cloud frame. PMPNet can effectively enlarge the receptive field of each pillar grid through iteratively aggregating messages on a $k$ NN graph. Then, an AST-GRU module composed of STA and TTA is presented to mine the spatiotemporal coherence in consecutive frames by using an attentive memory gating mechanism. The STA focuses on detecting the foreground objects, while the TTA aims to align the dynamic objects. Extensive experiments on the nuScenes benchmark have proved the better performance of our model. 


\section{References}

[1] Nicolas Ballas, Li Yao, Chris Pal, and Aaron Courville. Delving deeper into convolutional networks for learning video representations. In ICLR, 2016. 2, 4, 5

[2] Peter Battaglia, Razvan Pascanu, Matthew Lai, Danilo Jimenez Rezende, et al. Interaction networks for learning about objects, relations and physics. In NeurIPS, 2016. 3

[3] Joan Bruna, Wojciech Zaremba, Arthur Szlam, and Yann LeCun. Spectral networks and locally connected networks on graphs. In ICLR, 2014. 3

[4] Holger Caesar, Varun Bankiti, Alex H Lang, Sourabh Vora, Venice Erin Liong, Qiang Xu, Anush Krishnan, Yu Pan, Giancarlo Baldan, and Oscar Beijbom. nuscenes: A multimodal dataset for autonomous driving. arXiv preprint arXiv:1903.11027, 2019. 1, 2, 5, 7

[5] Xiaozhi Chen, Huimin Ma, Ji Wan, Bo Li, and Tian Xia. Multi-view 3d object detection network for autonomous driving. In $C V P R, 2017.2$

[6] Yilun Chen, Shu Liu, Xiaoyong Shen, and Jiaya Jia. Fast point r-cnn. In ICCV, 2019. 1, 2

[7] Kyunghyun Cho, Bart Van Merriënboer, Caglar Gulcehre, Dzmitry Bahdanau, Fethi Bougares, Holger Schwenk, and Yoshua Bengio. Learning phrase representations using rnn encoder-decoder for statistical machine translation. Conference on Empirical Methods in Natural Language Processing, 2014. 4,5

[8] Junyoung Chung, Caglar Gulcehre, KyungHyun Cho, and Yoshua Bengio. Empirical evaluation of gated recurrent neural networks on sequence modeling. arXiv preprint arXiv:1412.3555, 2014. 5

[9] Michaël Defferrard, Xavier Bresson, and Pierre Vandergheynst. Convolutional neural networks on graphs with fast localized spectral filtering. In NeurIPS, 2016. 3

[10] Lifeng Fan, Wenguan Wang, Siyuan Huang, Xinyu Tang, and Song-Chun Zhu. Understanding human gaze communication by spatio-temporal graph reasoning. In ICCV, 2019 . 3

[11] Andreas Geiger, Philip Lenz, and Raquel Urtasun. Are we ready for autonomous driving? the kitti vision benchmark suite. In CVPR, 2012. 1, 7

[12] Justin Gilmer, Samuel S Schoenholz, Patrick F Riley, Oriol Vinyals, and George E Dahl. Neural message passing for quantum chemistry. In ICML, 2017. 3

[13] Marco Gori, Gabriele Monfardini, and Franco Scarselli. A new model for learning in graph domains. In IEEE International Joint Conference on Neural Networks, 2005. 3

[14] Kaiming He, Xiangyu Zhang, Shaoqing Ren, and Jian Sun. Deep residual learning for image recognition. In $C V P R$, 2016. 5

[15] Mikael Henaff, Joan Bruna, and Yann LeCun. Deep convolutional networks on graph-structured data. arXiv preprint arXiv:1506.05163, 2015. 3

[16] Sepp Hochreiter and Jürgen Schmidhuber. Long short-term memory. Neural computation, 9(8):1735-1780, 1997. 4

[17] Jilin Hu, Jianbing Shen, Bin Yang, and Ling Shao. Infinitely wide graph convolutional networks: Semisupervised learning via gaussian processes. arXiv preprint arXiv:2002.12168, 2020. 3
[18] Peiyun Hu, Jason Ziglar, David Held, and Deva Ramanan. What you see is what you get: Exploiting visibility for $3 \mathrm{~d}$ object detection. In CVPR, 2020. 7

[19] Steven Kearnes, Kevin McCloskey, Marc Berndl, Vijay Pande, and Patrick Riley. Molecular graph convolutions: moving beyond fingerprints. Journal of computer-aided molecular design, 30(8):595-608, 2016. 3

[20] Diederik P Kingma and Jimmy Ba. Adam: A method for stochastic optimization. In ICLR, 2015. 7

[21] Jason Ku, Melissa Mozifian, Jungwook Lee, Ali Harakeh, and Steven L Waslander. Joint 3d proposal generation and object detection from view aggregation. In IROS, 2018. 2

[22] Jason Ku, Alex D Pon, and Steven L Waslander. Monocular $3 \mathrm{~d}$ object detection leveraging accurate proposals and shape reconstruction. In $C V P R, 2019.2$

[23] Qiuxia Lai, Wenguan Wang, Hanqiu Sun, and Jianbing Shen. Video saliency prediction using spatiotemporal residual attentive networks. TIP, 29:1113-1126, 2019. 5

[24] Alex H Lang, Sourabh Vora, Holger Caesar, Lubing Zhou, Jiong Yang, and Oscar Beijbom. Pointpillars: Fast encoders for object detection from point clouds. In CVPR, 2019. 1, 2, $3,4,7,8$

[25] Buyu Li, Wanli Ouyang, Lu Sheng, Xingyu Zeng, and Xiaogang Wang. Gs3d: An efficient 3d object detection framework for autonomous driving. In CVPR, 2019. 2

[26] Peiliang Li, Xiaozhi Chen, and Shaojie Shen. Stereo r-cnn based $3 \mathrm{~d}$ object detection for autonomous driving. In $C V P R$, 2019. 2

[27] Tao Li, Zhiyuan Liang, Sanyuan Zhao, Jiahao Gong, and Jianbing Shen. Self-learning with rectification strategy for human parsing. In $C V P R, 2020.3$

[28] Yujia Li, Daniel Tarlow, Marc Brockschmidt, and Richard Zemel. Gated graph sequence neural networks. In ICLR, 2016. 3

[29] Ming Liang, Bin Yang, Yun Chen, Rui Hu, and Raquel Urtasun. Multi-task multi-sensor fusion for $3 \mathrm{~d}$ object detection. In $C V P R, 2019.2$

[30] Ming Liang, Bin Yang, Shenlong Wang, and Raquel Urtasun. Deep continuous fusion for multi-sensor $3 \mathrm{~d}$ object detection. In $E C C V$, 2018. 2

[31] Tsung-Yi Lin, Priya Goyal, Ross Girshick, Kaiming He, and Piotr Dollár. Focal loss for dense object detection. In ICCV, 2017. 7

[32] Mason Liu and Menglong Zhu. Mobile video object detection with temporally-aware feature maps. In $C V P R, 2018$. 5

[33] Wenjie Luo, Bin Yang, and Raquel Urtasun. Fast and furious: Real time end-to-end $3 \mathrm{~d}$ detection, tracking and motion forecasting with a single convolutional net. In $C V P R, 2018$. $3,5,8$

[34] Fabian Manhardt, Wadim Kehl, and Adrien Gaidon. Roi10d: Monocular lifting of $2 \mathrm{~d}$ detection to $6 \mathrm{~d}$ pose and metric shape. In $C V P R, 2019.2$

[35] nuTonomy. nuscenes $3 \mathrm{~d}$ object detection challenge. https : //www. nuscenes.org/object-detection? externalData=all\&mapData=all\&modalities= Any /. 7

[36] Nanyun Peng, Hoifung Poon, Chris Quirk, Kristina Toutanova, and Wen-tau Yih. Cross-sentence n-ary relation extraction with graph 1stms. Transactions of the Association 
for Computational Linguistics, 5:101-115, 2017. 3

[37] Charles R Qi, Or Litany, Kaiming He, and Leonidas J Guibas. Deep hough voting for $3 \mathrm{~d}$ object detection in point clouds. In ICCV, 2019. 2

[38] Charles R Qi, Wei Liu, Chenxia Wu, Hao Su, and Leonidas J Guibas. Frustum pointnets for $3 \mathrm{~d}$ object detection from rgb-d data. In CVPR, 2018. 2

[39] Charles R Qi, Hao Su, Kaichun Mo, and Leonidas J Guibas. Pointnet: Deep learning on point sets for $3 \mathrm{~d}$ classification and segmentation. In CVPR, 2017. 2

[40] Siyuan Qi, Wenguan Wang, Baoxiong Jia, Jianbing Shen, and Song-Chun Zhu. Learning human-object interactions by graph parsing neural networks. In ECCV, 2018. 3

[41] Franco Scarselli, Marco Gori, Ah Chung Tsoi, Markus Hagenbuchner, and Gabriele Monfardini. The graph neural network model. IEEE Transactions on Neural Networks, 2008. 3

[42] Shaoshuai Shi, Xiaogang Wang, and Hongsheng Li. Pointr$\mathrm{cnn}$ : $3 \mathrm{~d}$ object proposal generation and detection from point cloud. In $C V P R, 2019.1,2$

[43] Xingjian Shi, Zhourong Chen, Hao Wang, Dit-Yan Yeung, Wai-Kin Wong, and Wang-chun Woo. Convolutional lstm network: A machine learning approach for precipitation nowcasting. In NeurIPS, 2015. 5

[44] Andrea Simonelli, Samuel Rota Bulo, Lorenzo Porzi, Manuel López-Antequera, and Peter Kontschieder. Disentangling monocular $3 \mathrm{~d}$ object detection. In ICCV, 2019. 7

[45] Leslie N Smith. Cyclical learning rates for training neural networks. In WACV, 2017. 7

[46] Xibin Song, Peng Wang, Dingfu Zhou, Rui Zhu, Chenye Guan, Yuchao Dai, Hao Su, Hongdong Li, and Ruigang Yang. Apollocar3d: A large 3d car instance understanding benchmark for autonomous driving. In CVPR, 2019. 1

[47] Ilya Sutskever, Oriol Vinyals, and Quoc V Le. Sequence to sequence learning with neural networks. In NeurIPS, 2014. 4

[48] Ashish Vaswani, Noam Shazeer, Niki Parmar, Jakob Uszkoreit, Llion Jones, Aidan N Gomez, Łukasz Kaiser, and Illia Polosukhin. Attention is all you need. In NeurIPS, 2017. 2

[49] Wenguan Wang, Xiankai Lu, Jianbing Shen, David J Crandall, and Ling Shao. Zero-shot video object segmentation via attentive graph neural networks. In ICCV, 2019. 5

[50] Wenguan Wang, Jianbing Shen, Ming-Ming Cheng, and Ling Shao. An iterative and cooperative top-down and bottom-up inference network for salient object detection. In CVPR, 2019. 5

[51] Wenguan Wang, Yuanlu Xu, Jianbing Shen, and Song-Chun Zhu. Attentive fashion grammar network for fashion landmark detection and clothing category classification. In CVPR, 2018. 3

[52] Wenguan Wang, Hailong Zhu, Jifeng Dai, Yanwei Pang, Jianbing Shen, and Ling Shao. Hierarchical human parsing with typed part-relation reasoning. In $C V P R, 2020.3$

[53] Xiaolong Wang, Ross Girshick, Abhinav Gupta, and Kaiming He. Non-local neural networks. In CVPR, 2018. 2

[54] Yan Wang, Wei-Lun Chao, Divyansh Garg, Bharath Hariharan, Mark Campbell, and Kilian Q Weinberger. Pseudo-lidar from visual depth estimation: Bridging the gap in $3 \mathrm{~d}$ object detection for autonomous driving. In CVPR, 2019. 2

[55] Yue Wang, Yongbin Sun, Ziwei Liu, Sanjay E Sarma,
Michael M Bronstein, and Justin M Solomon. Dynamic graph $\mathrm{cnn}$ for learning on point clouds. ACM Transactions on Graphics, 38(5):146, 2019. 4

[56] Yan Yan, Yuxing Mao, and Bo Li. Second: Sparsely embedded convolutional detection. Sensors, 18(10):3337, 2018. 2

[57] Bin Yang, Wenjie Luo, and Raquel Urtasun. Pixor: Realtime $3 \mathrm{~d}$ object detection from point clouds. In CVPR, 2018. 2

[58] Zetong Yang, Yanan Sun, Shu Liu, Xiaoyong Shen, and Jiaya Jia. Std: Sparse-to-dense 3d object detector for point cloud. In $I C C V, 2019.1,2$

[59] Yangyang Ye, Houjin Chen, Chi Zhang, Xiaoli Hao, and Zhaoxiang Zhang. Sarpnet: Shape attention regional proposal network for lidar-based 3d object detection. Neurocomputing, 379:53-63, 2020. 7

[60] Victoria Zayats and Mari Ostendorf. Conversation modeling on reddit using a graph-structured lstm. Transactions of the Association for Computational Linguistics, 6:121-132, 2018. 3

[61] Dingfu Zhou, Jin Fang, Xibin Song, Chenye Guan, Junbo Yin, Yuchao Dai, and Ruigang Yang. Iou loss for $2 \mathrm{~d} / 3 \mathrm{~d}$ object detection. In International Conference on $3 D$ Vision, 2019. 2

[62] Yin Zhou and Oncel Tuzel. Voxelnet: End-to-end learning for point cloud based 3d object detection. In CVPR, 2018. 1, $2,3,4,6,7$

[63] Benjin Zhu, Zhengkai Jiang, Xiangxin Zhou, Zeming Li, and Gang Yu. Class-balanced grouping and sampling for point cloud 3d object detection. arXiv preprint arXiv:1908.09492, 2019. 7

[64] Xizhou Zhu, Dazhi Cheng, Zheng Zhang, Stephen Lin, and Jifeng Dai. An empirical study of spatial attention mechanisms in deep networks. In ICCV, 2019. 2, 6

[65] Xizhou Zhu, Han Hu, Stephen Lin, and Jifeng Dai. Deformable convnets v2: More deformable, better results. In CVPR, 2019. 2, 6 